Secure and Effici ent Vehicle-to-Grid Energy Tradi ng in Oyber Physi cal Systems: An I nt egr at $\mathrm{i}$ on of Bl ockchai $\mathrm{n}$ and Edge Comput ing

\begin{tabular}{|l|l|}
\hline 著者 & $\begin{array}{l}\text { ZHOU Zhenyu, WANG Bi ngchen, DONG M anxi ong, } \\
\text { OTA Kaor u }\end{array}$ \\
\hline $\begin{array}{l}\text { j our nal or } \\
\text { publ i cat i on t i t l e }\end{array}$ & $\begin{array}{l}\text { I EEE Tr ansact i ons on Syst ens, Nan, and } \\
\text { Cyber net i cs: Syst ens }\end{array}$ \\
\hline vol une & 50 \\
\hline number & 1 \\
\hline page r ange & $43-57$ \\
\hline year & 2020 \\
\hline URL & ht t p: //hdl . handl e. net /10258/00010329 \\
\hline
\end{tabular}




\title{
Secure and Efficient Vehicle-to-Grid Energy Trading in Cyber Physical Systems: An Integration of Blockchain and Edge Computing
}

\author{
Zhenyu Zhou, Senior Member, IEEE, Bingchen Wang, Mianxiong Dong, Member, IEEE, \\ and Kaoru Ota, Member, IEEE
}

\begin{abstract}
Smart grid has emerged as a successful application of cyber-physical systems (CPSs) in the energy sector. Among numerous key technologies of the smart grid, vehicle to grid (V2G) provides a promising solution to reduce the level of demand-supply mismatch by leveraging the bidirectional energytrading capabilities of electric vehicles (EVs). In this paper, we propose a secure and efficient V2G energy trading framework by exploring blockchain, contract theory, and edge computing. First, we develop a consortium blockchain-based secure energy trading mechanism for V2G. Then, we consider the information asymmetry scenario, and propose an efficient incentive mechanism based on contract theory. The social welfare optimization problem falls into the category of difference of convex (DC) programming and is solved by using the iterative convex-concave procedure (CCP) algorithm. Next, edge computing has been incorporated to improve the successful probability of block creation. The computational resource allocation problem is modeled as a two-stage Stackelberg leader-follower game, and the optimal strategies are obtained by using the backward induction approach. Finally, the performance of the proposed framework is validated via numerical results and theoretical analysis.
\end{abstract}

Index Terms-cyber-physical system, consortium blockchain, contract theory, edge computing, vehicle-to-grid energy trading.

\section{INTRODUCTION}

\section{A. Background and Motivation}

$\mathbf{C}$ YBER-physical systems (CPSs) refer to a great variety of systems where the underlaying physical and computational components are implicitly integrated with each other to improve the adaptability, efficiency, reliability, and usability of physical systems [1], [2]. A typical application of CPSs in the energy sector is the smart grid [3], which employs upto-date information, communication, and control technologies to optimize the management and operation of power grids. Various researchers have investigated the smart grid from the perspective of cyber-physical integration such as cyberphysical energy management [4] and cyber-physical attack mitigation [5].

Manuscript received on June 10, 2018; revised on November 3, 2018.

This work is partially supported by JSPS KAKENHI Grant Numbers JP16K00117 and the KDDI Foundation.

M. Dong is the correspondence author.

Z. Zhou and B. Wang are with the State Key Laboratory of Alternate Electrical Power System with Renewable Energy Sources, School of Electrical and Electronic Engineering, North China Electric Power University, Beijing, China (e-mal: zhenyu_zhou@ncepu.edu.cn,wbc0203@163.com).

M. Dong and K. Ota are with the Department of Information and Electric Engineering, Muroran Institute of Technology, Muroran, Japan (e-mail: mx.dong@csse.muroran-it.ac.jp; ota@csse.muroran-it.ac.jp).
However, the large-scale penetration of intermittent distributed renewable energy sources and uncoordinated electric vehicles (EVs) leads to significant power fluctuation [6][9]. In order to maintain the reliable and safe operations of the smart grid, a large number of centralized generators and energy storage devices have to be deployed, which results in significant capital expenditure (CAPEX) and operational expenditure (OPEX) [10]. An alternative choice is to leverage the bidirectional energy-trading capabilities of EVs. Particularly, a large group of EVs can be coordinated to absorb excessive energy during the off-peak time and deliver energy back to the grid during the peak time, which provides a promising solution to flatten out the peak load and reduce the level of demandsupply mismatch without deploying additional generators and storage devices [11], [12]. This new energy trading paradigm is known as vehicle to grid (V2G), which is essential to build a safer and more sustainable CPS in the energy sector [13].

The studies on $\mathrm{V} 2 \mathrm{G}$ energy trading have received considerable attentions from both industry and academia. A distributed EV cooperation mechanism was proposed in [13], which not only enables the efficient management of charging and discharging operations, but also offers $\mathrm{V} 2 \mathrm{G}$ regulation services to support grid operation. In [14], Pal et al. presented a neighbor connection-based energy scheduling approach, which explores both vehicle-to-home and $\mathrm{V} 2 \mathrm{G}$ energy trading to reduce household electricity payments. Despite the abovementioned advantages, the wide area deployment of V2G still confronts several critical challenges, which are summarized as follows.

First, there lacks a distributed security mechanism for V2G energy trading. Conventional centralized mechanisms rely on a trusted intermediary to manage, audit, and verify every energy transaction [15], which is vulnerable to a series of security threats such as single point of failure, denial of service attacks, and privacy leakage [16]. For example, a transaction record may be changed, tampered, or deleted by some malicious middleman attackers. Most of the current works mainly concentrate on EV charging and discharging management [11], [13], [17], [18], while the security and privacy issues have been neglected. Therefore, a distributed security mechanism is indispensable to guarantee the reliable operation of large-scale V2G energy trading in an untrusted and nontransparent energy market.

Second, there lacks an efficient incentive mechanism for V2G energy trading. Due to the increased battery consumption 
and other costs incurred by discharging, EV owners are reluctant to participate in large-scale V2G energy trading unless they are well compensated. For example, EV owners usually suffer from the problem of range anxiety due to the limited cruising range and the long charging time [19]. Hence, when designing the incentive mechanism, a sufficient amount of energy must be preserved for EV owners in order to reach their destinations. Furthermore, the preference of each EV towards V2G participation belongs to the EV's private information, which is only known by the EV itself. This scenario is called information asymmetry. Most of the previous works, e.g., [11], [13], [20], have assumed that the EV's information is perfectly known by every entity in the energy market, and cannot be directly applied to V2G energy trading with asymmetric information. Therefore, it is of vital importance to develop an incentive mechanism, which can effectively maximize the economic benefits under the scenario of information asymmetry.

To address the above challenges, we develop a new V2G framework for CPSs, which leverages blockchain, contract theory, and edge computing to enable secure and efficient energy trading [21]. We consider a V2G energy trading scenario which involves three major entities: local energy aggregators (LEAGs), EVs, and edge computing service provider (ESP) [22], [23]. The energy trading between LEAGs and EVs is secured by employing consortium blockchain, in which all the transactions are created, propagated, and verified by authorized LEAGs. Then, we put forward a contract theorybased incentive mechanism to motivate EVs to participate in energy trading. The contract is tailored for the unique characteristic of each EV type to maximize the utility of the LEAG under the constraints of individual rationality (IR), incentive compatibility (IC), and monotonicity. The formulated contract optimization problem falls into the category of difference of convex (DC) programming. We further reduce the total number of IR and IC constraints by exploiting the relationships between adjacent EV types. The simplified problem is solved by using the iterative convex-concave procedure (CCP) algorithm. Several heuristic schemes are also developed as performance benchmarks. Next, edge computing has been incorporated in block creation to reduce transmission as well as processing latency. Specifically, LEAGs can purchase services from the ESP, and offload the computationintensive proof-of-work puzzles to proximate edge computing nodes. Considering the conflicting objectives of the ESP and LEAGs, the interaction between them is modeled as a twostage Stackelberg leader-follower game [24], [25], and the optimal service price and service demands are obtained by using the backward induction approach. Finally, we provide a comprehensive theoretical analysis on contract feasibility, performance convergence, game equilibrium, and energy trading security. The relationships among social welfare, EV type, reward, discharged electricity, edge computing service price, and edge computing service demands are elaborated via numerical results.

\section{B. Contributions}

The contributions of this work are summarized as follows:
- Consortium blockchain-based secure energy trading: We focus on typical electricity discharging scenario in V2G, and develop a consortium blockchain-based secure energy trading mechanism with moderate cost.

- Contract-based incentive mechanism design: To optimize the utility of LEAG under information asymmetry, we propose an efficient incentive mechanism for $\mathrm{V} 2 \mathrm{G}$ based on contract theoretical modeling.

- Edge computing-based task offloading: An edge computing-based task offloading mechanism is developed to increase the successful probability of block creation. The optimal pricing strategy of edge computing service is obtained by using Stackelberg game.

The remainder of this paper is organized as follows. Section II shows and compares some related works. Section III introduces the consortium blockchain for secure energy trading. Section IV presents the contract-based incentive mechanism. Section V elaborates the edge computing-based task offloading. Section VI shows the simulation results. Finally, Section VII concludes this paper.

\section{RELATED WORK}

Blockchain is a specific distributed shared database, which has been illustrated to possess salient advantages including security, immutability, and decentralization [15]. It allows every transaction to be recorded in a verifiable and permanent way, which is essential to create a distributed, transparent, and secure energy-trading environment. Blockchain has experienced rapid evolutions from version 1.0 to 3.0. While blockchain 1.0 and 2.0 are more related to Bitcoin and cryptcorrencies, as well as transferring contracts or properties, blockchain 3.0 extends its application fields from financial transactions to much broader sectors including energy, education, government, health, etc [26]. Recent works have employed blockchain to address the transaction security issues of peer-to-peer energy trading among EVs. Liu et al. proposed a decentralized blockchain-enabled EV charging scheme to simultaneously minimize the fluctuation level of the power grid and the EV charging cost [27]. In [28], Li et al. proposed a blockchain-based peer-to-peer energy trading system for industrial Internet of things, which relies on a credit-based payment strategy to reduce transaction confirmation latency. In [29], Aggarwal et al. proposed a blockchain model named EnergyChain to enable secure energy trading between smart grid and smart home, which involves miner selection, block creation and validation, and transaction handling. However, due to the high computational cost associated with block creation, blockchain has not been widely deployed in EVs with limited computational capabilities.

To reduce the computational complexity, we propose an edge computing-based consortium blockchain, in which a distributed ledger is created, publicly audited, and shared by multiple authorized nodes with moderate cost. First, unlike conventional blockchain, the distributed shared databases are only maintained by authorized LEAGs [30]. Second, edge computing [31], where computational resources are distributed across network edges, is employed to solve the proof-ofwork puzzles of block creation. Compared to centralized 
cloud computing, edge computing is more suitable to handle large-scale decentralized energy-trading transactions. Since the computational tasks are processed at close proximity to end users, dispensable network hops and transmission latency can be effectively eliminated.

Edge computing has been widely applied in a series of delay-sensitive applications to reduce computational delay. In [32], Garg et al. proposed a unmanned aerial vehicle (UAV)enabled edge computing framework where data are transferred from vehicles to edge nodes for real-time processing by leveraging UAVs as intermediate aerial communication devices. A probabilistic data structure (PDS)-based cyber-threat detection approach was developed to handle the data of vehicles. Zhou et al. proposed a new edge computing framework from an airground integration perspective, in which the communication and computational resources of both UAVs and vehicles are combined in a complementary manner to provide massiveconnectivity low-latency ultra-reliable services [33]. In [34], a robust mobile crowd sensing (RMCS) framework was developed by integrating deep learning based data validation with edge computing based local processing. Different from the above-mentioned works, we focus on the application scenario of blockchain-based energy trading in V2G, and the application-specific characteristics such as Hash power, successful probability of block creation, and propagation time of block are taken into account. The computational resource allocation problem is modeled as a two-stage Stackelberg leader-follower game, in which the positions of players are hierarchial, and the leader, i.e., the ESP, can enforce its own strategy upon the followers, i.e., LEAGs.

Another challenge in V2G energy trading is the incentive design. There exists some works which have addressed the incentive design problem in $\mathrm{V} 2 \mathrm{G}$ by exploiting robust optimization theory [11], Stackelberg game [35], genetic algorithm, [36], etc. A generalized Stackelberg game-based pricing scheme was proposed to optimize the operations of $\mathrm{V} 2 \mathrm{G}$ from the perspective of energy and reserve [35]. In [36], Ghofrani at al. developed a genetic algorithm-based EV charging and discharging mechanism to minimize the penalty cost of wind power imbalance as well as the EV discharging expenses. Nevertheless, all these works rely on a common assumption that the EV-side information is perfectly known by the LEAG, while information asymmetry has been neglected. In this work, we consider the more realistic scenario where the precise EV-side information is unknown, and develop a contract-based incentive mechanism to maximize the expected utility of LEAG based on contract theory. Contract theory provides a powerful tool to address the incentive problem with asymmetric information [37], and has already been widely applied in various applications including device-todevice communications [38], cognitive radio [37], and smallcell caching systems [39].

In summary, we investigate the feasibility of integrating consortium blockchain, contract theory, and edge computing to realize secure and efficient V2G energy trading in CPSs. The proposed framework can effectively improve transaction security and privacy protection, motivate EVs to participate in energy trading, and increase the successful probability of

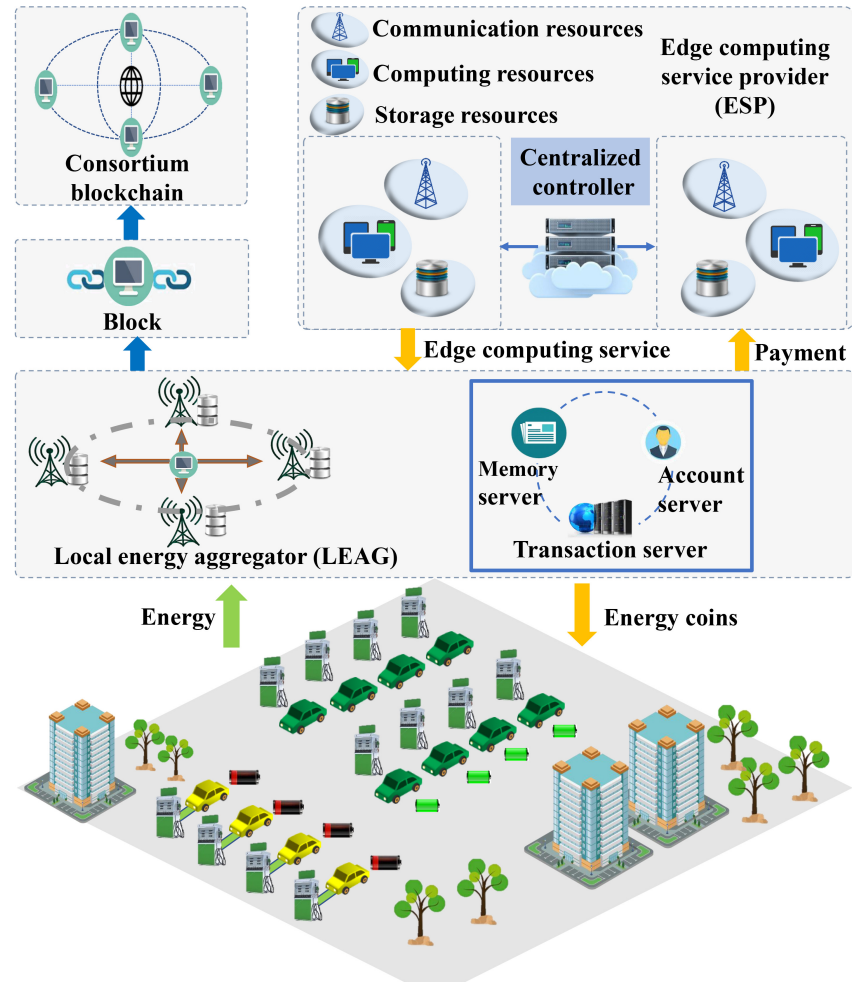

Fig. 1. Consortium blockchain-based secure energy trading for V2G.

block creation.

\section{CONSORTIUm BlockChain-BASEd SECURE ENERGY TRADING FRAMEWORK}

The consortium blockchain-based secure energy trading for V2G is shown in Fig. 1, which consists of three major entities: EV, LEAG, and ESP. The specific capability and functionality of each entity are elaborated as follows:

EV: EVs with bi-directional energy trading capabilities are able to play different roles. On the one hand, an EV can act as an energy producer by discharging its battery to provide electricity during the peak time. On the other hand, it can also act as an energy consumer by charging its battery with cheaper electricity while helping to absorb the excess energy during the off-peak time. With a properly designed incentive mechanism, each EV can actively adjust its charging and discharging behaviors to maximize its individual payoff. The details for how to design the incentive mechanism will be illustrated in Section IV-B.

LEAG: The LEAG provides an array of energy trading services including information collection, status monitoring, and charging/discharging coordination. For example, during the peak time, the LEAG can employ a group of discharging EVs to produce energy in response to local peak load demands. Meanwhile, the EVs which participate in the energy trading will obtain dedicated payments for their contributions to local supply-demand balance. Here, energy coin which is one kind of digital cryptocurrency is utilized as the payment for the energy trading. 
There are three major components in the LEAG: a memory server, an account server, and a transaction server. All of the transaction records in the consortium blockchain are stored in the memory server. The digital assets of each EV in terms of energy coins are stored in a digital wallet. To preserve privacy, the true address of the wallet is replaced by a set of public keys, e.g., random pseudonyms. Each EV also has a transaction account which stores all of its transaction records. The mapping relationships between random wallet addresses and the corresponding transaction account are maintained in the account server. The transaction server is responsible to implement incentive mechanisms and coordinate charging and discharging activities.

ESP: The ESP with unified control of the integrated computation, communication, and storage resources provides edge computing services for LEAGs. The ESP issues a price for its service, and each LEAG determines the service demand to be purchased based on the price. Then, the computation-intensive proof-of-work puzzles can be offloaded from a LEAG to its proximate edge computing nodes instead of being processed locally or by remote cloud nodes. The details for how to model the interactions between the ESP and LEAGs, and how to design the optimal service price and service demands will be illustrated in subsection V.

The operation details of the consortium blockchain-based secure energy trading are explained as follows. Existing cryptographic algorithms including elliptic curve digital signature, Boneh-Boyen short signature, and SHA-256, have been employed. In the beginning, each EV has to register with a legitimate authority to obtain its public key, privacy key, and certificate. The public/privacy keys can be generated and distributed by the authority. The certificate represents a unique identity for the EV via binding its registration information. Each EV has a set of wallet addresses issued by the authority. During initialization, an EV finds the wallet address that is used by its nearest LEAG and verifies the wallet integrity. Afterwards, it downloads the corresponding data from the memory server.

The secrecy of the private key is maintained by the EV for signing transactions, while the public key is shared with other authorized entities to verify its signatures [40]. The public/privacy keys can be generated by using some specific algorithms such as elliptic curve digital signature algorithm (ECDSA) [41], a new signature scheme based on lattice [42], a novel anti-quantum transaction authentication scheme in the blockchain [43], etc.

After energy trading, a discharging EV will receive the specified reward if the corresponding contract item has been successfully fulfilled. The energy coins are transferred from the LEAG to the wallet address of the EV. The transaction is digitally signed by the LEAG to ensure its integrity and authenticity, and then is broadcasted to the network. The other LEAGs not only check the transaction via analyzing the digital signature, but also check whether the LEAG which issues this transaction is authorized to spend the energy coin or not. A fake transaction will be discarded, and only valid transactions are included in a new block.

Next, to add the new block to the blockchain, all the authorized LEAGs start the mining process, in which they compete to find a valid proof of work similar to Bitcoin [44]. This process requires to find a random value $\alpha$, combined with the hash of the previous block header $\Phi$ and the hash of transactions, which satisfies $\operatorname{Hash}(\alpha+\Phi)<\beta$. Here, $\beta$ represents the level of difficulty [45]. If a LEAG finds a a valid proof of work, it will broadcast the result to other authorized LEAGs. Upon receiving the result, the other LEAGs verify it and determine whether to accept it or not. If a majority of LEAGs agree on the result, i.e., a consensus has been reached, then the new block will be added to the blockchain, and the LEAG which created this block will be rewarded by a certain amount of energy coins. Finally, the amount of energy coins that is transferred from the LEAG to the EV will be received in the EV's wallet.

It requires extremely high computational power to create a false block, find a valid proof of work before other LEAGs, and control the majority of the LEAGs [26]. Furthermore, malicious attacks which try to modify the transaction records can be prevented since each validated block is linked to the previous block via secure cryptography methods. Any change of a transaction will affect both the block containing it and subsequent blocks.

If the computational capability of a LEAG is limited, it can purchase edge computing services from the ESP. Then, the computation-intensive proof-of-work process is handled by nearby edge computing nodes with powerful computation capabilities, and the successful probability of block creation will be significantly improved. We assume that transaction verification is handled locally by LEAGs in order to reduce the cost of purchasing computing service. The reason is the computational complexity of transaction verification is much lower that that of block creation, i.e., mining. Taking Bitcoin for example, the level of difficulty is dynamically adjusted after every 2,016 blocks to guarantee that the block generation time is exactly 600 seconds [46]. As the total computational power of the overall network increases continuously, the difficulty is also increased accordingly to maintain the fixed block generation time. Nevertheless, the proposed framework can be easily extended to the scenario where both block creation and transaction verification are offloaded to edge computing nodes.

The data shared among EVs, LEAGs, and ESP also suffer from the chosen-plaintext attack (CPA) and chosen-ciphertext attack (CCA). CPA represents an attack strategy for cryptanalysis in which the ciphertexts for arbitrary plaintexts are available to the the attacker [47]. CCA represents another attack model in which the cryptanalyst collect information from decryptions of chosen ciphertexts and try to recover the secret key based on the collected information. Since how to mitigate CPA and CCA has already been intensively investigated, it is ignored here due to space limitation and is left to the future work. interested readers can refer to related works [47]-[50].

The decentralized consensus can be guaranteed due to the following two reasons. First, the ESP mainly makes a revenue by selling computing services, and is not rewarded for block creation. Thus, it does not have a strong incentive to cheat in verification. Second, a new block is verified by every LEAG 
independently according to the same rules. If some dishonest LEAGs accept some fake transactions in a new block, the other honest LEAGs will update their own copy of the blockchain without using the invalid block. As a result, the fake block branch is much shorter than the valid branch, which will be discarded since LEAGs only maintain a copy of the longest chain [51].

\section{A Contract-BASEd InCEntive Mechanism For V2G ENERGY TRADING}

\section{A. EV Type Modeling}

We use EV type to quantify the preference of an EV towards discharging, which is only known by the EV itself. A highertype $\mathrm{EV}$ is more willing to participate in the $\mathrm{V} 2 \mathrm{G}$ energy trading and discharge a larger amount of electricity to gain a higher reward. It is intuitive that EVs with higher types are more preferred by the LEAG. For the sake of simplicity, we assume that the set of EV types belongs to a discrete and finite space. The EV type is defined as follows.

Definition 1 : Considering a parking lot with $K$ discharging EVs, these EVs can be sorted in an ascending order based on their preferences and classified into $K$ types. If the set of EV types is denoted as $\Theta=\left\{\theta_{1}, \cdots, \theta_{k}, \cdots, \theta_{K}\right\}$, then we have

$$
\theta_{1}<\cdots<\theta_{k}<\cdots<\theta_{K}, \quad k=1, \cdots, K .
$$

In the following, we derive the specific expression of the $\mathrm{EV}$ type. Considering type $\theta_{k} \mathrm{EV}$, the stage of charge (SoC) is calculated as [52]

$$
S o C_{k}^{c}=\frac{E_{k}^{c}}{E_{k, \max }}
$$

where $E_{k}^{c}$ represents the amount of currently available energy, and $E_{k, \max }$ is the battery capacity. After discharging, the remaining SoC should satisfy the minimum energy requirement of traveling, which is given by

$$
\frac{E_{k}^{c}-L_{k}}{E_{k, \max }} \geq \chi\left(d_{k}\right),
$$

where $L_{k}$ is the required amount of electricity, and $d_{k}$ is the distance that has to be traveled before the next charging. $\chi\left(d_{k}\right)$ denotes the minimum electricity required to travel the distance $d_{k}$, which is a monotonically increasing function of $d_{k}$. By combining (2) and (3), we can derive the discharging capability, which is given by

$$
L_{k} \leq\left[S o C_{k}^{c}-\chi\left(d_{k}\right)\right] E_{k, \max } .
$$

Hence, type $\theta_{k}$ can be defined as

$$
\theta_{k}:=\left[S o C_{k}^{c}-\chi\left(d_{k}\right)\right] E_{k, \max } .
$$

Remark 1. From (5), it is observed that $\theta_{k}$ is positively proportional to $S o C_{k}^{c}$ and $E_{k, \max }$, and inversely proportional to $\chi\left(d_{k}\right)$. For example, a larger EV type represents that either the EV has more energy available, or it will not travel a long distance in the near future.

In the information asymmetry scenario, the LEAG does not know the specific type of each EV, but only has the knowledge of the probability distribution of each type. We assume that the
LEAG knows that there are a total of $K$ types of discharging EVs and an EV belongs to type $\theta_{k}$ with a probability $P_{k}$, i.e., $\sum_{k=1}^{K} P_{k}=1$.

\section{B. Contract Formulation}

Instead of providing the same contract for EVs with different types, a contract which consists of $K$ contract items is designed for $K$ types of discharging EVs, i.e., one for each type. For example, the contract item designed for type $\theta_{k}$ $\mathrm{EV}$ is denoted as $\left(L_{k}, R_{k}\right)$, where $L_{k}$ denotes the required electricity, and $R_{k}$ is the dedicated reward in terms of energy coins. The contract is defined as $\mathcal{C}=\left\{\left(L_{k}, R_{k}\right), \forall k \in \mathcal{K}\right\}$, where $\mathcal{K}=\{1, \cdots, k, \cdots, K\}$.

Considering the $K$ types of EVs, the expected utility of the LEAG is calculated as

$$
U_{L}\left(\left\{L_{k}\right\},\left\{R_{k}\right\}\right)=K \sum_{k=1}^{K} P_{k}\left(\gamma_{L} L_{k}-R_{k}\right),
$$

where $\gamma_{L}$ is the unit value of electricity for the LEAG.

Remark 2. A contract item $\left(L_{k}=0, R_{k}=0\right)$ means that type $\theta_{k} \mathrm{EV}$ does not intend to participate in discharging. On the other hand, the LEAG will benefit from the EV discharging only if $\gamma_{L} L_{k}-R_{k} \geq 0$. Otherwise, the LEAG has no incentive to employ type $\theta_{k} \mathrm{EV}$ for discharging.

The utility function of type $\theta_{k}$ EV which accepts the contract item $\left(L_{k}, R_{k}\right)$ is given by

$$
U_{k}^{E V}\left(L_{k}, R_{k}\right)=\theta_{k} m\left(R_{k}\right)-\gamma L_{k}
$$

where $\gamma$ is the unit cost of discharging the battery. $\theta_{k} m\left(R_{k}\right)$ represents the value of $R_{k}$ for type $\theta_{k} \mathrm{EV}$. The function $m\left(R_{k}\right)$ is a monotonically increasing concave function of $R_{k}$, where $m(0)=0, m^{\prime}\left(R_{k}\right)>0$, and $m^{\prime \prime}\left(R_{k}\right)<0$. Without loss of generality, $m\left(R_{k}\right)$ can be defined as a quadratic function, i.e.,

$$
m\left(R_{k}\right)=-\frac{a}{2} R_{k}^{2}+b R_{k},
$$

where $a$ and $b$ are assumed as constants, which should satisfy $m^{\prime}\left(R_{k}\right)>0$ and $m^{\prime \prime}\left(R_{k}\right)<0$. Nevertheless, the proposed scheme can be extended to other forms.

The expected social welfare is the total sum utility of the LEAG and the $K \mathrm{EVs}$, which is given by

$$
\begin{aligned}
S W\left(\left\{L_{k}\right\},\left\{R_{k}\right\}\right) & =U_{L}\left(\left\{L_{k}\right\},\left\{R_{k}\right\}\right) \\
& +K \sum_{k=1}^{K} P_{k} U_{k}^{E V}\left(L_{k}, R_{k}\right) .
\end{aligned}
$$

The utility of LEAG maximization problem under asymmetric information is formulated as

$$
\begin{array}{ll}
\text { P1 : } & \max _{\left\{L_{k}\right\},\left\{R_{k}\right\}} U_{L}\left(\left\{L_{k}\right\},\left\{R_{k}\right\}\right) \\
\text { s.t. } \quad C_{1}: \theta_{k} m\left(R_{k}\right)-\gamma L_{k} \geq 0,(I R) \\
\quad C_{2}: \theta_{k} m\left(R_{k}\right)-\gamma L_{k} \geq \theta_{k} m\left(R_{k^{\prime}}\right)-\gamma L_{k^{\prime}},(I C) \\
C_{3}: 0 \leq R_{1}<\cdots<R_{k}<\cdots<R_{K} \\
C_{4}: L_{k} \leq \theta_{k} \\
\quad \forall k, k^{\prime} \in \mathcal{K}
\end{array}
$$


where $C_{1}, C_{2}$, and $C_{3}$ represent the IR, IC, and monotonicity constraints, respectively. $C_{4}$ represents the upper bound of $L_{k}$.

Definition 2 : The IR, IC, and monotonicity constraints are defined as follows:

- Individual rationality (IR) constraint: Type $\theta_{k} \mathrm{EV}$, $\forall k \in \mathcal{K}$, will get a nonnegative payoff if it selects the contract item $\left(L_{k}, R_{k}\right)$.

- Incentive compatibility (IC) constraint: The IC constraint ensures the self-revealing property of the contract. For instance, type $\theta_{k} \mathrm{EV}, \forall k \in \mathcal{K}$, will get the maximum payoff if and only if it selects the contract item $\left(L_{k}, R_{k}\right)$ designed for its own type.

- Monotonicity constraint: The reward of type $\theta_{k} \mathrm{EV}$, $\forall k \in \mathcal{K}$, should be higher than that of type $\theta_{k-1} \mathrm{EV}$, and lower than that of type $\theta_{k+1} \mathrm{EV}$.

Based on the IR, IC, and monotonicity constraints, the following properties can be derived.

Lemma 1. For any $k, k^{\prime} \in \mathcal{K}$, if $\theta_{k}>\theta_{k^{\prime}}$, then $R_{k}>R_{k^{\prime}}$. $R_{k}=R_{k^{\prime}}$ if and only if $\theta_{k}=\theta_{k^{\prime}}$.

Lemma 2. For any $L_{k}, R_{k} \in \mathcal{C}$, the following inequalities hold

$$
\begin{aligned}
& 0 \leq R_{1} \leq \cdots \leq R_{k} \leq \cdots \leq R_{K}, \\
& 0 \leq L_{1} \leq \cdots \leq L_{k} \leq \cdots \leq L_{K}, \\
& 0 \leq U_{1}^{E V} \leq \cdots \leq U_{k}^{E V} \leq \cdots \leq U_{K}^{E V} .
\end{aligned}
$$

Proof: The detailed proof of Lemma 1 and Lemma 2 are omitted here due to space limitation. A similar proof can be found in [38].

\section{Optimal Contract Design under Information Asymmetry}

1) Contract Feasibility: First, we define the sufficient and necessary conditions for contract feasibility.

Theorem 1. Contract feasibility: The contract $\mathcal{C}=$ $\left\{\left(L_{k}, R_{k}\right), \forall k \in \mathcal{K}\right\}$ is feasible if and only if all the following conditions are satisfied:

- $0 \leq R_{1} \leq \cdots \leq R_{k} \leq \cdots \leq R_{K}$ and $0 \leq L_{1} \leq \cdots \leq$ $L_{k} \leq \cdots \leq L_{K}$

- $\theta_{1} m\left(R_{1}\right)-\gamma L_{1} \geq 0$

- For any $k \in\{2, \cdots, K\}, \gamma L_{k-1}+\theta_{k-1}\left[m\left(R_{k}\right)-\right.$ $\left.m\left(R_{k-1}\right)\right] \leq \gamma L_{k} \leq \gamma L_{k-1}+\theta_{k}\left[m\left(R_{k}\right)-m\left(R_{k-1}\right)\right]$.

Proof: The detailed proof of Theorem 1 is omitted here due to space limitation. A similar proof can be found in Appendix D of [37].

2) Problem Transformation: The utility of LEAG maximization problem $\mathbf{P} \mathbf{1}$ involves $K$ IR constraints and $K(K-1)$ IC constraints. To provide a tractable solution, the following procedures are carried out to simplify the problem.

Step 1: IR Constraints Elimination

For type $\theta_{k} \mathrm{EV}, k \in \mathcal{K}, k \neq 1$, we can derive

$$
\theta_{k} m\left(R_{k}\right)-\gamma L_{k} \geq \theta_{k} m\left(R_{1}\right)-\gamma L_{1}>\theta_{1} m\left(R_{1}\right)-\gamma L_{1} \geq 0,
$$

where the first inequality is due to the IC constraint, the second inequality is due to $\theta_{k}>\theta_{1}$, and the third inequality is due to the IR constraint. Hence, if the IR constraint of type $\theta_{1} \mathrm{EV}$ is guaranteed, then the IR constraints of EVs with higher types are automatically satisfied.

\section{Step 2: IC Constraints Elimination}

We define the IC constraints between type $\theta_{k}$ and type $\theta_{k^{\prime}}, k^{\prime} \in\{1, \cdots, k-1\}$, as downward incentive constraints (DICs). Similarly, the IC constraints between type $\theta_{k}$ and type $\theta_{k^{\prime}}, k^{\prime} \in\{k+1, \cdots, K\}$, are defined as upward incentive constraints (UICs). In the following, we will show that both the DICs and UICs can be reduced.

We consider three adjacent EV types, i.e., $\theta_{k-1}<\theta_{k}<$ $\theta_{k+1}$, which satisfy

$$
\begin{array}{r}
\theta_{k+1} m\left(R_{k+1}\right)-\gamma L_{k+1} \geq \theta_{k+1} m\left(R_{k}\right)-\gamma L_{k}, \\
\theta_{k} m\left(R_{k}\right)-\gamma L_{k} \geq \theta_{k} m\left(R_{k-1}\right)-\gamma L_{k-1},
\end{array}
$$

where (13) denotes the DIC between type $\theta_{k+1}$ and type $\theta_{k}$, and (14) denotes the DIC between type $\theta_{k}$ and $\theta_{k-1}$.

By combining $R_{k+1} \geq R_{k} \geq R_{k-1}$, we have

$$
\theta_{k+1} m\left(R_{k+1}\right)-\gamma L_{k+1} \geq \theta_{k+1} m\left(R_{k-1}\right)-\gamma L_{k-1} .
$$

Therefore, if the DIC between type $\theta_{k+1}$ and $\theta_{k}$ holds, then the DIC between $\theta_{k+1}$ and $\theta_{k-1}$ also holds. The DIC constraints can be extended downward from type $\theta_{k-1}$ to type $\theta_{1}$, which are given by

$$
\begin{aligned}
\theta_{k+1} m\left(R_{k+1}\right)-\gamma L_{k+1} & \geq \theta_{k+1} m\left(R_{k-1}\right)-\gamma L_{k-1}, \\
& \geq \cdots \\
& \geq \theta_{k+1} m\left(R_{1}\right)-\gamma L_{1} .
\end{aligned}
$$

Thus, we demonstrate that if the DICs between adjacent types hold, then all the DICs hold automatically. Similarly, we can demonstrate that if the UICs between adjacent types hold, then all the UICs hold automatically.

Based on the above analysis, the $K$ IR constraints and $K(K-1)$ IC constraints can be reduced to 1 and $K-1$, respectively. $\mathbf{P} \mathbf{1}$ is rewritten as

$$
\begin{array}{ll}
\text { P2: } & \max _{\left\{L_{k}\right\},\left\{R_{k}\right\}} U_{L}\left(\left\{L_{k}\right\},\left\{R_{k}\right\}\right), \\
\text { s.t. } & C_{1}: \theta_{1} m\left(R_{1}\right)-\gamma L_{1} \geq 0,(I R) \\
& C_{2}: \theta_{k} m\left(R_{k-1}\right)-\gamma L_{k-1} \leq \theta_{k} m\left(R_{k}\right)-\gamma L_{k}, \quad(I C) \\
& C_{3}, C_{4}, k=2, \cdots, K .
\end{array}
$$

3) Optimal Contract with Reduced Constraints: We can prove that the objective of $\mathbf{P} \mathbf{2}$ is a concave function by checking the Hessian matrix. However, convex programming cannot be directly applied here because the constraint $C_{2}$ involves the difference of two concave functions, i.e., $\theta_{k} m\left(R_{k-1}\right)-\gamma L_{k-1}$ and $\theta_{k} m\left(R_{k}\right)-\gamma L_{k}$. Therefore, the CCP algorithm proposed in [53] is adopted to solve P2, which is summarized in Algorithm 1.

Denote $f_{k}\left(R_{k}\right)=\theta_{k} m\left(R_{k}\right)$. Since $f_{k}\left(R_{k}\right)$ is differentiable with regards to $R_{k}, f_{k}\left(R_{k}\right)$ can be approximated by using its first-order Taylor series expansion as

$$
f_{k}\left(R_{k}\right) \approx f_{k}\left(R_{k, 0}[\tau]\right)+\nabla f_{k}\left(R_{k, 0}[\tau]\right)\left(R_{k}-R_{k, 0}[\tau]\right),
$$

where $R_{k, 0}[\tau]$ represents the initial point at iteration $\tau$. 
Hence, the constraint $C_{2}$ with the difference of two concave functions is transformed to the difference of a concave function and an affine function, which is written as

$$
\begin{aligned}
\tilde{C}_{2}: & \theta_{k} m\left(R_{k-1}\right)-\gamma L_{k-1} \\
& \leq f_{k}\left(R_{k, 0}[\tau]\right)+\nabla f_{k}\left(R_{k, 0}[\tau]\right)\left(R_{k}-R_{k, 0}[\tau]\right) .
\end{aligned}
$$

By replacing $C_{2}$ with $\tilde{C}_{2}, \mathbf{P 2}$ is transformed into a convex programming problem, and can be easily solved by using Karush-Kuhn-Tucker (KKT) conditions. At each iteration $\tau$, the local optimal solutions $\hat{L}_{k}[\tau]$ and $\hat{R}_{k}[\tau]$ are obtained by solving the transformed convex problem. Then, the initial point for Taylor series expansion at iteration $\tau+1$ is defined as $R_{k, 0}[\tau+1]=\hat{R}_{k}[\tau]$. Next, the above iteration is repeated to derive a new local optimal solution.

The iterative process terminates until a predefined stopping criterion is satisfied. For example, the improvement in the social welfare is less than or equal to some positive threshold $\epsilon$, i.e.,

$U_{L}\left(\left\{\hat{L}_{k}[\tau+1]\right\},\left\{\hat{R}_{k}[\tau+1]\right\}\right)-U_{L}\left(\left\{\hat{L}_{k}[\tau]\right\},\left\{\hat{R}_{k}[\tau]\right\}\right) \leq \epsilon$.

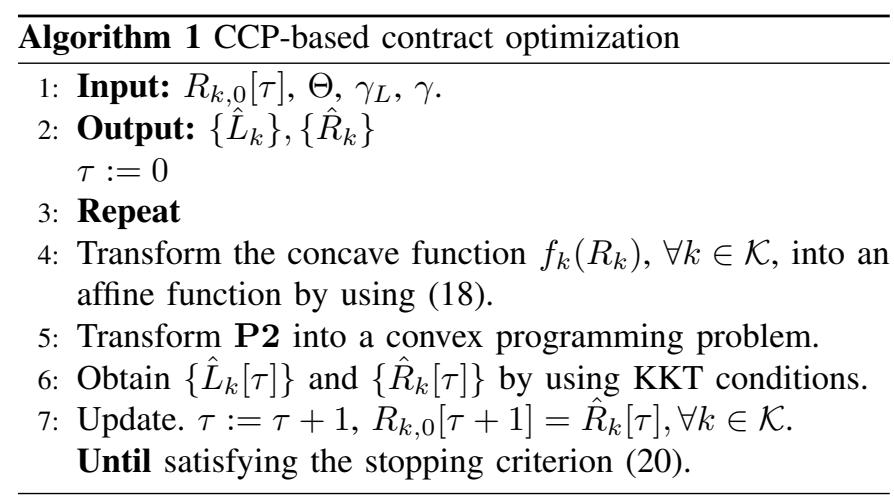

Theorem 2. Convergence: At any iteration $\tau$, the obtained $\left\{\hat{L}_{k}[\tau]\right\}$ and $\left\{\hat{R}_{k}[\tau]\right\}$ are feasible. Furthermore, $\{S W\}_{\tau=0}^{\infty}$ is nondecreasing, and will converge to the maximum social welfare, i.e.,

$$
U_{L}\left(\left\{\hat{L}_{k}[\tau]\right\},\left\{\hat{R}_{k}[\tau]\right\}\right) \leq U_{L}\left(\left\{\hat{L}_{k}[\tau+1]\right\},\left\{\hat{R}_{k}[\tau+1]\right\}\right) .
$$

Proof: The detailed proof of Theorem 2 is omitted here. A similar proof can be found in [53] .

\section{Optimal Contract Design without Information Asymmetry}

If there exists a selfish LEAG which is precisely aware of each EV's type, it can further increase its profit as long as each EV only accepts the contract item designed for its own type. In this scenario, the LEAG has to ensure that the payoff of each $\mathrm{EV}$ is non-negative. Otherwise, the EVs have no incentive to accept the contract item. To this end, the contract item has to meet the IR constraint. Furthermore, the contract item has to satisfy the following property:

Lemma 3. In the contract design without information asymmetry, any contract item $\left(L_{k}, R_{k}\right) \in \mathcal{C}$ should satisfy $\theta_{k} m\left(R_{k}\right)=\gamma L_{K}$. That is, the payoff for any EV is zero.
Proof: Lemma 3 can be proved by contradiction. Given an optimal contract item $\left(R_{k}, L_{k}\right)$, if $\theta_{k} m\left(R_{k}\right)-\gamma L_{k}>0$, then the LEAG can increase its utility by increasing $L_{k}$ until $\theta_{k} m\left(R_{k}\right)-\gamma L_{k}=0$. This contradicts with the assumption that $\left(L_{k}, R_{k}\right)$ is optimal.

Thus, by enforcing the utility of each EV to be zero, the social welfare is equivalent to the utility of the LEAG. The corresponding optimization problem is formulated as

$$
\begin{array}{ll}
\text { P3 : } & \max _{\left\{L_{k}\right\},\left\{R_{k}\right\}} U_{L}\left(\left\{L_{k}\right\},\left\{R_{k}\right\}\right), \\
\text { s.t. } & C_{1}: \theta_{k} m\left(R_{k}\right)-\gamma L_{k}=0, \\
& C_{2}: 0 \leq R_{1}<\cdots<R_{k}<\cdots<R_{K}, \\
& C_{3}, C_{4}, \forall k \in \mathcal{K} .
\end{array}
$$

To solve (22), we have to work out the solutions of $K$ quadratic equations, i.e., $\theta_{k} m\left(R_{k}\right)-\gamma L_{k}=0, \forall k \in \mathcal{K}$. Assuming that $R_{k 1}$ and $R_{k 2}$ are the two solutions of the $k$-th quadratic equation, the optimal solution is given by

$\left(\left\{L_{k}\right\},\left\{R_{k}\right\}\right)=$

$\underset{\left\{R_{k}\right\} \in\left(\left\{R_{k 1}\right\},\left\{R_{k 2}\right\}\right)}{\arg \max }\left(U_{L}\left(\left\{L_{k 1}\right\},\left\{R_{k 1}\right\}\right), U_{L}\left(\left\{L_{k 2}\right\},\left\{R_{k 2}\right\}\right)\right)$.

Lemma 4. In the contract design without information asymmetry, for any EV type $\theta_{k}, k \in \mathcal{K}, R_{k}$ is fixed regardless of $\theta_{k}$.

Proof: Substituting $\theta_{k} m\left(R_{k}\right)-\gamma L_{k}=0$ into (9), it can be verified that the social welfare $S W$ increases monotonically with $\sum_{k=1}^{K} L_{k}$. Hence, the LEAG can increase $L_{k}$ until $L_{k}=$ $\theta_{k}$. Next, substituting $L_{k}=\theta_{k}$ into $\theta_{k} m\left(R_{k}\right)-\gamma L_{k}=0$, we have $m\left(R_{k}\right)=\gamma_{L}$, which means that $R_{k}$ is fixed regardless of $\theta_{k}$.

\section{Edge Computing-BAsed Computational TASK OFFLOADING}

\section{A. Hierarchical Game Formulation}

In order to win the block mining competition, a LEAG can purchase edge computing services from the ESP to enlarge its computational capability. We assume that there are $N$ LEAGs, and the set of LEAGs is denoted as $\mathcal{N}$. The service demand of the $n$-th LEAG is denoted as $s_{n}$. For the $n$-th LEAG, the successful probability of block creation, i.e., $P_{n}$, depends on two factors: its relative hash power $P_{n, h}$ [54] and its block orphaning probability $P_{n, o}$ [44], which are explained as follows.

The relative hash power of the $n$-th LEAG is defined as the ratio of its computational power with respect to the total computational power, i.e.,

$$
P_{n, h}\left(s_{n}\right)=\frac{s_{n}}{s_{n}+\sum_{n^{\prime} \in \mathcal{N}, n^{\prime} \neq n} s_{n^{\prime}}}, n \in \mathcal{N},
$$

where $P_{n, h}>0$ and $\sum_{j \in \mathcal{N}} P_{j, h}=1$.

Upon finding a valid proof-of-work, the $n$-th LEAG has to broadcast the created block to the other LEAGs in order to reach a consensus. If the $n$-th LEAG happens to choose a large block which propagates slowly due to the data size, 
then the block is more likely to be discarded due to the high transmission latency. Accordingly, the chance of the $n$ th LEAG to win the competition of block mining will be diminished. This phenomenon is called orphaning [44]. By assuming that the block propagation time follows a Poisson distribution, the orphaning probability is given by

$$
P_{n, o}=1-e^{-\frac{\Delta t\left(D_{n}\right)}{T}},
$$

where $T$ denotes the expected block interval time, which is 10 mins in Bitcoin. $\Delta t\left(D_{n}\right)$ denotes the relative propagation time of a block with size $D_{n}$, which is defined as

$$
\Delta t\left(D_{n}\right)=t\left(D_{n}\right)-t(0) \text {. }
$$

Here, $t\left(D_{n}\right)$ is the propagation time to deliver a block with size $D_{n}$, and $t(0)$ presents the lag of the communication channel, i.e., the time required for delivering the block header. $t(0)$ is bounded by the constraint $t(0) \geq d_{c} / c$, where $d_{c}$ denotes the transmission distance, and $c$ denotes the speed of light.

Based on the approach proposed in [44], $t\left(D_{n}\right)$ can be approximated by using its first-order Taylor series expansion around $D_{n}=0$ as

$$
t\left(D_{n}\right) \approx t(0)+\left.\nabla t\right|_{D_{n}=0} D_{n},
$$

where the second term of (27) is partially related to the carrying capacity of the communication channel. Based on the Shannon-Hartley theorem, it can be written as $\left.\nabla t\right|_{D_{n}=0}=$ $1 /\left(G_{1} G_{2}\right)$, where $G_{1}$ and $G_{2}$ present the channel capacity and coding gain, respectively. Hence, by taking $\left.\nabla t\right|_{D_{n}=0}=$ $1 /\left(G_{1} G_{2}\right)$ and (27) into (26), $\Delta t\left(D_{n}\right)$ is written as

$$
\Delta t\left(D_{n}\right)=t\left(D_{n}\right)-t(0) \approx \frac{D_{n}}{G_{1} G_{2}} .
$$

The successful probability of block creation, i.e., $P_{n}$, is given by

$$
\begin{aligned}
P_{n}\left(s_{n}\right) & =P_{n, h}\left(s_{n}\right)\left(1-P_{n, o}\right) \\
& =\frac{s_{n}}{s_{n}+\sum_{n^{\prime} \in \mathcal{N}, n^{\prime} \neq n} s_{n^{\prime}}} e^{-\frac{D_{n}}{G_{1} G_{2} T}} .
\end{aligned}
$$

Once the consensus process is successful, the $n$-th LEAG will gain a revenue which consists of two parts: the reward for its contribution to block creation $Q_{n}$, and the transaction fee $M_{n}$. The net revenue of the $n$-th LEAG can be calculated as the expected profit minus the service cost, i.e.,

$$
U_{n, b}\left(s_{n}\right)=\left(Q_{n}+M_{n}\right) P_{n}\left(s_{n}\right)-p_{c} s_{n},
$$

where $p_{c}$ is the unit service price of edge computing.

The utility of the ESP is defined as the total revenue obtained from service provisioning minus the operation cost, i.e.,

$$
U_{E}\left(p_{c}\right)=\sum_{n \in \mathcal{N}} p_{c} s_{n}-\gamma_{c} \sum_{n \in \mathcal{N}} s_{n},
$$

where $\gamma_{c}$ is the unit cost of service provisioning.

Owing to the dominant market position of the ESP compared to LEAGs, the competitive interaction between the ESP and LEAGs can be modeled as a two-stage Stackelberg leaderfollower game. In the first stage, the ESP is the leader that determines the unit service price $p_{c}$, and obtains the revenue from LEAGs for solving the offloaded proof-of-work puzzles. In the second stage, the LEAGs act as the followers, and determine the service demand to be purchased. The two-stage Stackelberg leader-follower game is formulated as follows:

\section{Stage 1. the service price optimization problem:}

$$
\begin{array}{ll}
\mathbf{P} 4: & \max _{p_{c}} U_{E}\left(p_{c}\right), \\
\text { s.t. } & C_{5}: p_{c, \text { min }} \leq p_{c} \leq p_{c, \max },
\end{array}
$$

where $p_{c, \min }$ and $p_{c, \max }$ denote the minimum and maximum bounds of the unit service price.

\section{Stage 2. the service demand optimization problem:}

$$
\begin{aligned}
& \text { P5 : } \max _{s_{n}} U_{n, b}\left(s_{n}\right), \\
& \text { s.t. } \quad C_{6}: s_{n, \min } \leq s_{n} \leq s_{n, \max },
\end{aligned}
$$

where $s_{n, \min }$ is the minimum computational resources (hash power) required by the $n$-th LEAG, and $s_{n, \max }$ represents the maximum computational resources that can be provided by the ESP.

\section{B. Equilibrium Analysis}

The optimal price and the optimal service demands can be derived by using the backward induction approach [24].

1) The Solution of the Second-Stage Optimization Problem: First, given a service price $p_{c}$, the second-stage service demand optimization problem P5 is solved for each LEAG. During the service demand optimization, every LEAG competes with each other to maximize its own relative hash power, and thus, to maximize its successful probability of block creation. From (24), we can observe that the relative hash power of the $n$-th LEAG not only depends on its strategy $s_{n}$, but also depends on the strategies of the other LEGAs, e.g., $s_{n^{\prime}}, n^{\prime} \in \mathcal{N}, n^{\prime} \neq n$. Therefore, the competition among $N$ LEAGs can be modeled as a $N$-player noncooperative game. Denote the optimal strategy of the $n$-th LEAG as $s_{n}^{*}$, and let $\mathbf{s}_{-n}^{*}=\left\{s_{n^{\prime}}, n^{\prime} \in \mathcal{N}, n^{\prime} \neq n\right\}$ represent the set of optimal strategies of the other LEAGs in the set $N$ excluding the $n$-th LEAG. We have the following properties:

Theorem 3. Nash equilibrium: The set of optimal service demand strategies, i.e., $\left\{s_{n}^{*}, \mathbf{s}_{-n}^{*}\right\}, \forall n \in \mathcal{N}$, constitutes a Nash equilibrium of the second-stage $N$-player noncooperative game.

Proof: For any feasible $s_{n}, n \in \mathcal{N}$, we have

$$
U_{n, b}\left(s_{n}^{*}, \mathbf{s}_{-n}^{*}, p_{c}\right) \geq U_{n, b}\left(s_{n}, \mathbf{s}_{-n}^{*}, p_{c}\right) .
$$

Hence, $\left\{s_{n}^{*}, \mathbf{s}_{-n}^{*}\right\}$ constitutes a Nash equilibrium.

Theorem 4. Nash equilibrium existence: A Nash equilibrium exists in the second-stage $N$-player noncooperative game.

Proof: Based on [55], a Nash equilibrium exists if the following two conditions are satisfied:

- $\left\{s_{n}, n \in \mathcal{N}\right\}$ is a nonempty compact convex subset of a Euclidean space. 
- $U_{n, b}\left(s_{n}\right)$ is continuous and quasi concave with regards to $s_{n}$.

First, for any $n \in \mathcal{N}$, the strategy space $\left[s_{n, \min }, s_{n, \max }\right]$ is a convex, continuous, compact and non-empty subset of the Euclidean space, which satisfies the first condition.

Second, the second-order derivative of (30) with regards to $s_{n}$ is given by

$$
\frac{\partial^{2} U_{n, b}}{\partial s_{n}^{2}}=\left(Q_{n}+M_{n}\right) \frac{\partial^{2} P_{n, b}}{\partial s_{n}^{2}} e^{-\frac{D_{n}}{C G T}}<0,
$$

where

$$
\frac{\partial^{2} P_{n, h}}{\partial s_{n}^{2}}=-2 \frac{\sum_{n^{\prime} \in \mathcal{N}, n^{\prime} \neq n} s_{n^{\prime}}}{\left(s_{n}+\sum_{n^{\prime} \in \mathcal{N}, n^{\prime} \neq n} s_{n^{\prime}}\right)^{3}}<0 .
$$

This proves that $U_{n, b}\left(s_{n}\right)$ is concave with regards to $s_{n}$. Therefore, a Nash equilibrium exists in the second-stage $N$ player noncooperative game.

Theorem 5. Best response: Given $\mathrm{s}_{-n}^{*}$, the best response function of the $n$-th LEAG, i.e., $B_{n}\left(\mathbf{s}_{-n}^{*}\right)$, is given by (38).

Proof: Since $U_{n, b}\left(s_{n}\right)$ is concave with regards to $s_{n}$ and $C_{6}$ is affine, $\mathbf{P 5}$ is a standard convex programming problem. By setting the first-order derivative of (30) to zero, i.e., $\frac{\partial U_{n, b}}{\partial s_{n}}=0$, we have

$$
\hat{s}_{n}=\sqrt{\frac{\left(Q_{n}+M_{n}\right) \sum_{n^{\prime} \in \mathcal{N}, n^{\prime} \neq n} s_{n^{\prime}}}{p_{c} e^{\frac{D_{n}}{G_{1} G_{2} T}}}}-\sum_{n^{\prime} \in \mathcal{N}, n^{\prime} \neq n} s_{n^{\prime}} .
$$

The optimal solution $s_{n}^{*}$ can be obtained as

$$
s_{n}^{*}=B_{n}\left(\mathbf{s}_{-n}^{*}\right)=\left\{\begin{array}{lc}
s_{n, \min }, & \hat{s}_{n}<s_{n, \min } \\
\hat{s}_{n}, & s_{n, \min } \leq \hat{s}_{n} \leq s_{n, \max } \\
s_{n, \max }, & \hat{s}_{n}>s_{n, \max }
\end{array}\right.
$$

Theorem 6. Nash equilibrium uniqueness: If the condition

$$
\frac{2(N-1) e^{\frac{D_{n}}{G_{1} G_{2} T}}}{Q_{n}+M_{n}}<\sum_{n \in \mathcal{N}} \frac{e^{\frac{D_{n}}{G_{1} G_{2} T}}}{Q_{n}+M_{n}}
$$

is satisfied, then the Nash equilibrium of the second-stage $N$ player noncooperative game is unique.

Proof: The Nash equilibrium is unique if the best response function of any LEAG, e.g., $B_{n}\left(\mathbf{s}_{-n}^{*}\right), \forall n \in \mathcal{N}$, is a standard function. Based on [55], $B_{n}\left(\mathbf{s}_{-n}^{*}\right)$ is a standard function if the following conditions are satisfied:

- Positivity: $B_{n}\left(\mathbf{s}_{-n}^{*}\right)>0$.

- Monotonicity: If $\mathbf{s}_{-n}^{*} \geq \tilde{\mathbf{s}}_{-n}^{*}$, then $B_{n}\left(\mathbf{s}_{-n}^{*}\right) \geq B_{n}\left(\tilde{\mathbf{s}}_{-n}^{*}\right)$. - Scalability: For all $\psi>1, \psi B_{n}\left(\mathbf{s}_{-n}^{*}\right)>B_{n}\left(\psi \mathbf{s}_{-n}^{*}\right)$.

First, to prove positivity, we have to prove that

$$
B_{n}\left(\mathbf{s}_{-n}^{*}\right)=\mathbf{s}_{-n}^{*}>0 \text {. }
$$

By setting $\frac{\partial U_{n, b}}{\partial s_{n}}=\left(Q_{n}+M_{n}\right) \frac{\partial P_{n, h}}{\partial s_{n}} e^{-\frac{D_{n}}{G_{1} G_{2} T}}-p_{c}=0$, we can obtain

$$
\frac{\sum_{n^{\prime} \in \mathcal{N}, n^{\prime} \neq n} s_{n^{\prime}}}{\left(\sum_{n \in \mathcal{N}} s_{n}\right)^{2}}=\frac{p_{c} e^{\frac{D_{n}}{G_{1} G_{2} T}}}{Q_{n}+M_{n}}
$$

Take the summation of all miners, i.e., $\sum_{n \in \mathcal{N}}$, for both sides of (41), we have

$$
\frac{(N-1) \sum_{n \in \mathcal{N}} s_{n}}{\left(\sum_{n \in \mathcal{N}} s_{n}\right)^{2}}=\sum_{n \in \mathcal{N}} \frac{p_{c} e^{\frac{D_{n}}{G_{1} G_{2} T}}}{Q_{n}+M_{n}}
$$

which can be written as

$$
\sum_{n \in \mathcal{N}} s_{n}=\frac{N-1}{\sum_{n \in \mathcal{N}} \frac{p_{c} e^{\frac{D_{n}}{G_{1} G_{2} T}}}{Q_{n}+M_{n}}}
$$

Besides, from (41), we can derive the expression of $\sum_{n \in \mathcal{N}} s_{n}$ as

$$
\sum_{n \in \mathcal{N}} s_{n}=\sqrt{\frac{\left(Q_{n}+M_{n}\right) \sum_{n^{\prime} \in \mathcal{N}, n^{\prime} \neq n} s_{n^{\prime}}}{p_{c} e^{\frac{D_{n}}{G_{1} G_{2} T}}} .}
$$

By substituting (43) into (44) and utilizing the condition (39), we have

$$
\sum_{n^{\prime} \in \mathcal{N}, n^{\prime} \neq n} s_{n^{\prime}}=\left(\frac{N-1}{\sum_{n \in \mathcal{N}} \frac{p_{c} e^{\frac{D_{n}}{G_{1} G_{2} T}}}{Q_{n}+M_{n}}}\right)^{2} \frac{p_{c} e^{\frac{D_{n}}{G_{1} G_{2} T}}}{Q_{n}+M_{n}} .
$$

By taking the square of both sides of (39), and multiplying both sides with $p_{c}$, we get the expression

$$
\left(\frac{N-1}{\sum_{n \in \mathcal{N}} \frac{p_{c} e^{\frac{D_{n}}{G_{1}} T}}{Q_{n}+M_{n}}}\right)^{2} \frac{p_{c} e^{\frac{D_{n}}{G_{1} G_{2} T}}}{Q_{n}+M_{n}}<\frac{Q_{n}+M_{n}}{4 p_{c} e^{\frac{D_{n}}{G_{1} G_{2} T}}} .
$$

Substituting (46) into (45), we have

$$
\sum_{n^{\prime} \in \mathcal{N}, n^{\prime} \neq n} s_{n^{\prime}}<\frac{Q_{n}+M_{n}}{4 p_{c} e^{\frac{D_{n}}{G_{1} G_{2} T}}}<\frac{Q_{n}+M_{n}}{p_{c} e^{\frac{D_{n}}{G_{1} G_{2} T}} .}
$$

Multiplying both sides of (47) with $\sum_{n^{\prime} \in \mathcal{N}, n^{\prime} \neq n} s_{n^{\prime}}$ and taking the square root, we can derive that

$$
\sum_{n^{\prime} \in \mathcal{N}, n^{\prime} \neq n} s_{n^{\prime}}<\sqrt{\frac{\left(Q_{n}+M_{n}\right) \sum_{n^{\prime} \in \mathcal{N}, n^{\prime} \neq n} s_{n^{\prime}}}{p_{c} e^{\frac{D_{n}}{G_{1} G_{2} T}}} .}
$$

Thus, the positivity condition can be proved as

$$
s_{n}^{*}=\sqrt{\frac{\left(Q_{n}+M_{n}\right) \sum_{n^{\prime} \in \mathcal{N}, n^{\prime} \neq n} s_{n^{\prime}}}{p_{c} e^{\frac{D_{n}}{G_{1} G_{2} T}}}}-\sum_{n^{\prime} \in \mathcal{N}, n^{\prime} \neq n} s_{n^{\prime}}>0 .
$$

Next, we prove that the response function $B_{n}$ is monotone. If $\tilde{\mathbf{s}}_{-n}<\mathbf{s}_{-n}$, then the expression of $B_{n}\left(\mathbf{s}_{-n}-B_{n}\left(\tilde{\mathbf{s}}_{-n}\right)\right)$ is provided in (50). If (50) is positive, then both the two functions of the right side, i.e., $g_{1}$ and $g_{2}$, should also be positive. Since $\tilde{\mathbf{s}}_{-n}<\mathbf{s}_{-n}$, it can be easily proved that $g_{1}>0$. By using $g_{1}>0, g_{2}$ can be written as

$$
g_{2}>\sqrt{\frac{Q_{n}+M_{n}}{p_{c} e^{\frac{D_{n}}{G_{1} G_{2} T}}}}-2 \sqrt{\sum_{n^{\prime} \in \mathcal{N}, n^{\prime} \neq n} s_{n^{\prime}}} .
$$




$$
\begin{aligned}
& B_{n}\left(\mathbf{s}_{-n}\right)-B_{n}\left(\tilde{\mathbf{s}}_{-n}\right)=\sqrt{\frac{Q_{n}+M_{n}}{p_{c} e^{\frac{D_{n}}{G_{1} G_{2} T}}}}\left(\sqrt{\sum_{n^{\prime} \in \mathcal{N}, n^{\prime} \neq n} s_{n^{\prime}}}-\sqrt{\sum_{n^{\prime} \in \mathcal{N}, n^{\prime} \neq n} \tilde{s}_{n^{\prime}}}\right)-\left(\sum_{n^{\prime} \in \mathcal{N}, n^{\prime} \neq n} s_{n^{\prime}}-\sum_{n^{\prime} \in \mathcal{N}, n^{\prime} \neq n} \tilde{s}_{n^{\prime}}\right)
\end{aligned}
$$

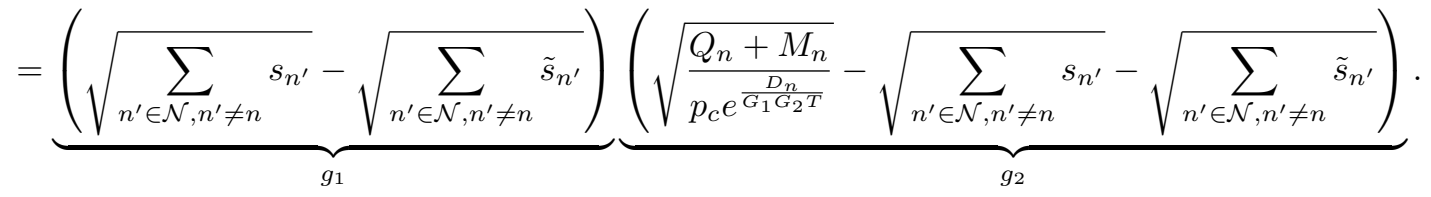

By taking the square root of both sides of (47), we have

$$
g_{2}>\sqrt{\frac{Q_{n}+M_{n}}{p_{c} e^{\frac{D_{n}}{G_{1} G_{2} T}}}}-2 \sqrt{\sum_{n^{\prime} \in \mathcal{N}, n^{\prime} \neq n} s_{n^{\prime}}}>0 .
$$

Thus, we have $B_{n}\left(\mathbf{s}_{-n}\right)-B_{n}\left(\tilde{\mathbf{s}}_{-n}\right)=g_{1} g_{2}>0$. This completes the proof of monotonicity.

Finally, We will prove the scalability of (38) as follows:

$$
\begin{aligned}
& \psi B_{n}\left(\mathbf{s}_{-n}^{*}\right)-B_{n}\left(\psi \mathbf{s}_{-n}^{*}\right)= \\
&(\psi-\sqrt{\psi}) \sqrt{\frac{\left(Q_{n}+M_{n}\right) \sum_{n^{\prime} \in \mathcal{N}, n^{\prime} \neq n} s_{n^{\prime}}}{p_{c} e^{\frac{D_{n}}{G_{1} G_{2} T}}}>0 .}
\end{aligned}
$$

Hence, the best response function of any LEAG is a standard function, and the Nash equilibrium is unique.

2) The Solution of the First-Stage Optimization Problem: Based on the optimal service demand strategies of all the LEAGs obtained in the second stage, the first-stage service price optimization problem can be solved. By substituting the Nash equilibrium of the second-stage $N$-player noncooperative game into (31), the utility of the ESP $U_{E}$ can be written as

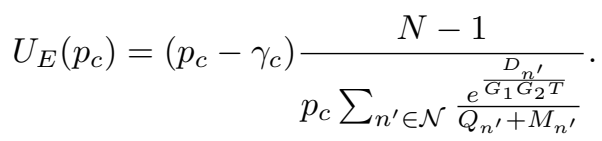

Then, we have the following properties:

Theorem 7. Concavity: P4 is a standard convex optimization problem.

Proof: We can prove that the second order derivative of (54) is negative, which is given by

$$
\frac{\partial U_{E}}{\partial p_{c}}=-2 \frac{\gamma_{c}(N-1)}{p_{c}^{3} \sum_{n \in \mathcal{N}} \frac{e^{\frac{D_{n}}{G_{1} G_{2} T}}}{Q_{n}+M_{n}}}<0 .
$$

This complete the proof.

Since P4 is a convex optimization problem, the optimal solution $p_{c}^{*}$ can be easily solved by using KKT conditions. We have the following property:

Theorem 8. Stackelberg equilibrium: The Nash equilibrium of the second-stage $N$-player noncooperative game $\left\{s_{n}^{*}, \mathbf{s}_{-n}^{*}\right\}$ and the optimal solution of the first-stage service price optimization problem $p_{c}^{*}$ constitute the Stackelberg equilibrium.

Proof: For any feasible $s_{n}, n \in \mathcal{N}$, we have

$$
U_{n, b}\left(s_{n}^{*}, \mathbf{s}_{-n}^{*}, p_{c}^{*}\right) \geq U_{n, b}\left(s_{n}, \mathbf{s}_{-n}^{*}, p_{c}^{*}\right) .
$$

Furthermore, for any feasible $p_{c}$, we have

$$
U_{E}\left(s_{n}^{*}, \mathbf{s}_{-n}^{*}, p_{c}^{*}\right) \geq U_{E}\left(s_{n}^{*}, \mathbf{s}_{-n}^{*}, p_{c}\right) .
$$

This completes the proof.

\section{Simulation RESUlts AND ANALYSis}

In this section, we validate the proposed scheme via simulations.

\section{A. Contract Feasibility and Social Welfare}

We consider a parking lot with one LEAG and $K=20$ EVs. We assume that the EV type follows a Gaussian distribution. For any $\mathrm{EV}$, the battery capacity is $24 \mathrm{kWh}$, and the unit discharging cost is 10 cents/kWh, i.e., $\gamma=10$. The unit revenue of electricity for the LEAG is 13 cents $/ \mathrm{kWh}$, i.e., $\gamma_{L}=13$. The contract without information asymmetry and the linear-pricing scheme studied in [38] are utilized for the purpose of comparison. In the linear-pricing scheme, the LEAG does not distinguish EVs by their types, and offers a unified price $\rho$ to all the EVs. There exists a linear relationship between the reward and discharged electricity, e.g., $R_{k}=\rho L_{k}$, $k=1, \cdots, K$.

Fig. 2(a) and Fig. 2(b) show the discharged electricity and the reward, respectively. It is observed that both the reward and the discharged electricity increase monotonically with the EV type, which follows Lemma 2. Furthermore, numerical results show that the contract without information asymmetry demands much higher amounts of electricity from EVs, but offers EVs with the same reward, which is consistent with Lemma 4. In the linear-pricing scheme, the amount of discharged electricity is lower than the other schemes, which represents that the unified pricing scheme cannot effectively motivate EVs to participate in the energy trading.

Fig. 2(c) shows the relationship between utilities of type 4 , type 8 , and type $12 \mathrm{EV}$ and contract items. It is validated that the proposed contract is incentive compatible. An EV can achieve its maximum utility if and only if it selects the contract item dedicated for its type. Furthermore, we can observe that the utility of EV increases with the EV type, which is explained in Lemma 2.

Fig. 3(a) and Fig. 3(b) show the utility of the LEAG and the utilities of EVs, respectively. Without information asymmetry, the LEAG can achieve a much higher utility, while the utility of any EV remains zero, which is consistent of Lemma 3. Thus, the presence of information asymmetry actually protects EVs from over-exploitation since the precise knowledge of 


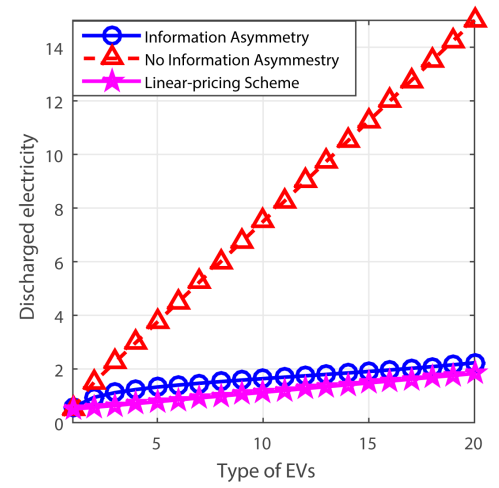

(a)

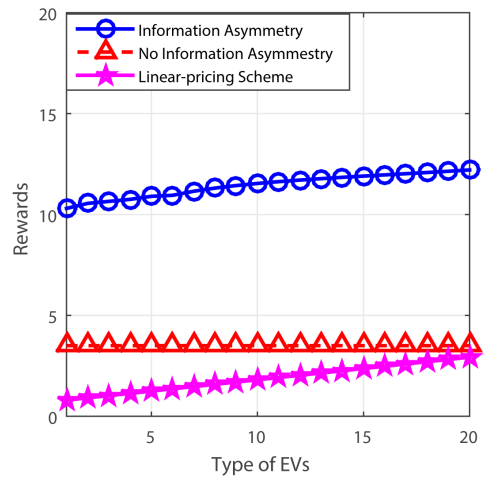

(b)

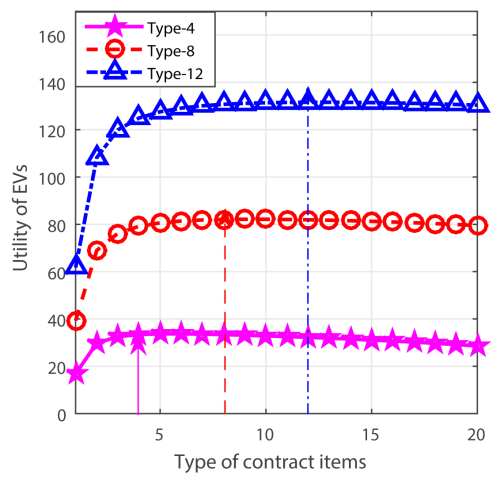

(c)

Fig. 2. Contract feasibility: (a) discharged electricity; (b) reward; (c) EV's utility.

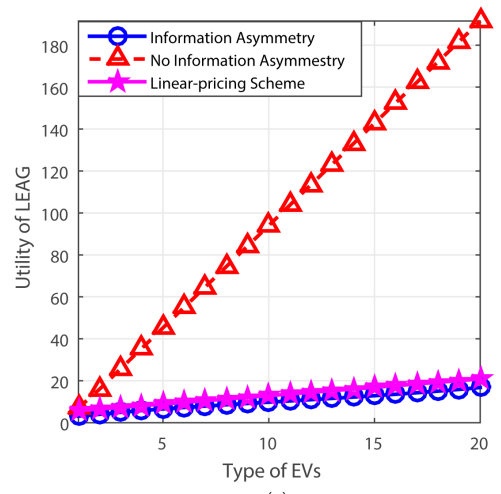

(a)

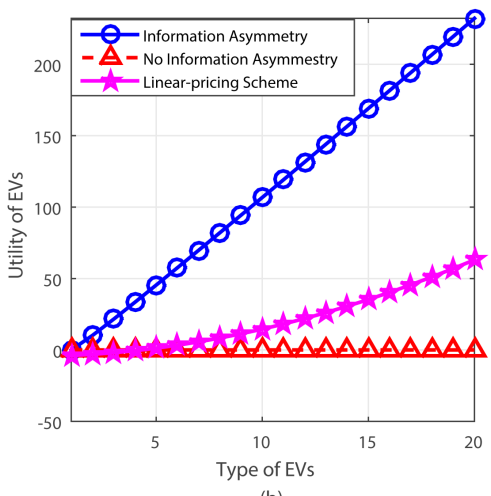

(b)

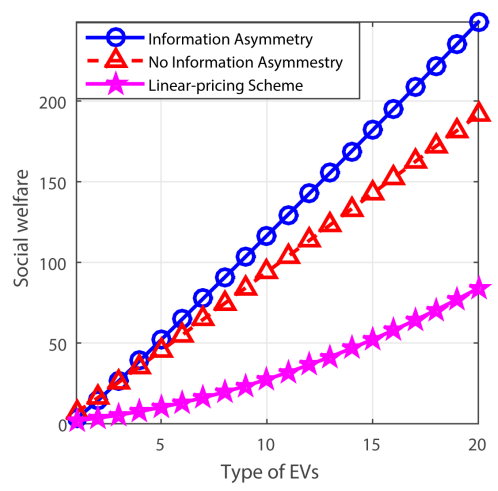

Fig. 3. System performance: (a) LEAG's utility; (b) EV's utility; (c) social welfare.

EV type is unknown to the LEAG. The linear-pricing scheme achieves the worst performance since the type of EV has not been fully exploited.

Fig. 3(c) shows the relationship between social welfare and EV type. The contract without information asymmetry performs worse than the contract with information asymmetry. The reason is that under complete information, the utility of any EV is exactly zero, which significantly decreases the social welfare. The linear-pricing scheme achieves the lowest social welfare because the information of EV type has been neglected.

Fig. 4 shows the convergence performance of the proposed CCP-based solution. Three initial points, i.e., $\left\{R_{k, 0}[1]\right\}=$ 12,10 , and 9 are chosen to characterize the impact of initial point on the convergence speed. As the iteration number increases, all the three cases converge to the optimal social welfare. Particularly, the case with $\left\{R_{k, 0}[1]\right\}=12$ only requires 10 iterations to reach convergence. The reason is that 12 is closest to the average value of the optimal rewards shown in Fig. 2(b) (which is 12.66). In comparison, the case with $\left\{R_{k, 0}[1]\right\}=9$ requires more than 200 iterations.

\section{B. Edge Computing-based Computational Task Offloading}

To verify the benefits brought by edge computing, we consider two cases with four and eight LEAGs, i.e., $N=4$ and $N=8$, respectively. In the case of $N=4$, we assume that edge computing service is not available to the first three LEAGs, and we fix their computational power as 10, 20, and 30, respectively. Meanwhile, we assume that the fourth LEAG can purchase service from the ESP and vary its purchased service demand to demonstrate the impact on the successful probability of block creation. In the case of $N=8$, the computational power of the first seven LEAGs is fixed in the range $[10,40]$. The conventional scheme without the assistance of edge computing is used for comparison [30]. We assume that $D_{n}$ follows a normal distribution, i.e., $\mathcal{N}\left(\mu_{D_{n}}, \sigma_{D_{n}}^{2}\right)$, where $\mu_{D_{n}}=200$ and $\sigma_{D_{n}}^{2}=10$.

Fig. 5 shows the successful probability versus the purchased service demand. When the service demand purchased from the ESP is 55, simulation results demonstrate that the successful probability of the proposed edge computing-based scheme outperforms that of the conventional scheme by $92.4 \%$ and $124.6 \%$ in the cases of $N=4$ and $N=8$, respectively. The reason is that the relative computational power of the LEAG which has access to edge computing services can be increased by orders of magnitudes compared to those LEAGs which only rely on their local computational power.

Fig. 6 shows the total service demand, the profit of the ESP, and the average profit of the LEAG versus the transaction fee $M_{n}$. The simulation parameters are $s_{n, \min }=90, s_{n, \max }=$ 


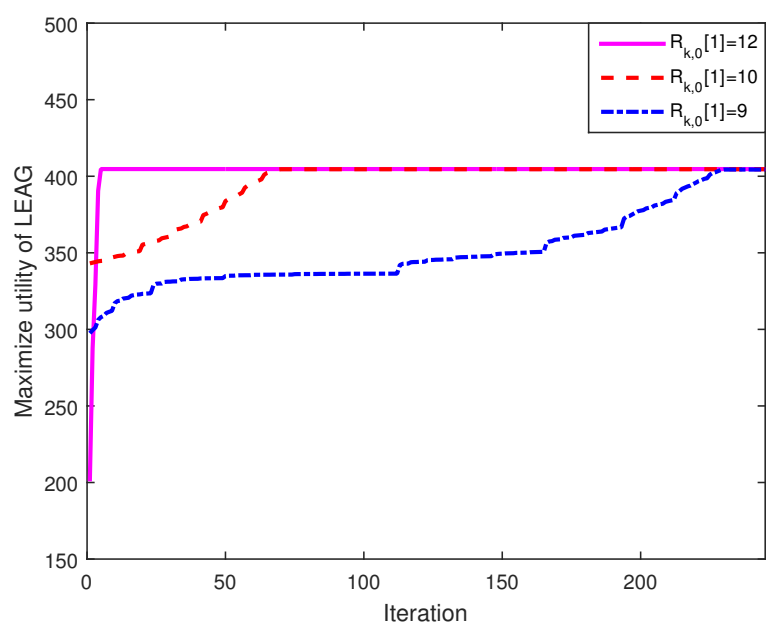

Fig. 4. The convergence performance of the proposed CCP-based solution.

$210, p_{c, \min }=0, p_{c, \max }=15, Q_{n}=12000, \gamma_{c}=3, G_{1}=50$, $G_{2}=4$, and $N=50$. Fig. 6 (a) and (b) demonstrate that both the total service demand and the profit of the ESP increase monotonically with the transaction fee. The reason behind is that the increased transaction fee provides a larger incentive for LEAGs to purchase more services from the ESP. This not only improves the successful probability of LEAGs, but also increases the profit of the ESP. Furthermore, it is observed that both the total service demand and the profit of the ESP increase monotonically with the block creation reward $Q_{n}$, which is also due to the fact that a higher reward provides a greater motivation for LEAGs to buy more services.

Fig. 6 (c) shows the average profit of the $n$-th LEAG achieved by four different schemes: the proposed scheme, the conventional scheme without edge computing, the aggressive scheme in which the $n$-th LEAG always purchases the maximum amount of services $s_{n, \max }$, and the conservative scheme in which the $n$-th LEAG always purchases the minimum amount of services $s_{n, \min }$. It is clear that the proposed scheme outperforms the other three heuristic schemes because the LEAG's strategy is optimized with regards to the transaction fee. When the transaction fee is low, the conservative scheme performs better than the aggressive scheme. It is not worthy to buy more services because the expected profit cannot compensate the cost of purchasing services. In comparison, when the transaction fee is high enough, the LEAG should purchase a larger amount of services to increase the chance of winning because the expected profit is much higher than the service cost. Under all scenarios, the conventional scheme without edge computing performs the worst due to the reason explained in Fig. 5.

\section{Privacy and Security Analysis}

In this subsection, we provide the privacy and the security analysis.

Anonymity: Instead of using its true identity, each EV uses a unique public key to communicate with others, which prevents malicious attackers from tracking an EV's identity.

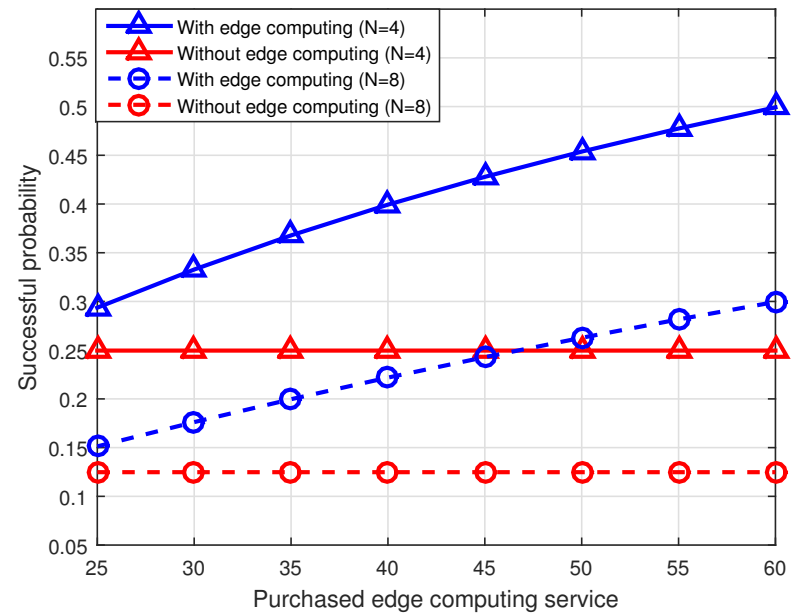

Fig. 5. Successful probability versus purchased amount of services.

Furthermore, an EV can change its public key after each transaction to avoid the linking attack, i.e., the different pieces of data belonging to the same EV are linked together to deanonymize the EV.

Authentication: In the process of proof-of-work, every transaction has to be publicly audited and authenticated by authorized LEAGs. It is impossible to compromise all of the authorized LEAGs in the network.

Integrity: Once a block has been appended into the blockchain, it contains the hash of the previous block, and its own hash will be contained in the subsequent block. Therefore, it is infeasible to modify the block unless the majority of the computational power are controlled by a malicious attacker. Moreover, the transaction data contained in a block are encrypted with asymmetric encryption techniques. It takes a tremendous cost to decrypt the encrypted data without knowing the private key.

Transparency: Since the blockchain technology is open source, any user, software developer, and service provider can have access to the blockchain and monitor the corresponding transaction data. That is, the transaction data are not saved in one single node and are transparent to all entities. As a result, any malicious data modification can be noticeable and traceable.

\section{CONCLUSIONS}

In this paper, we proposed a secure and efficient V2G energy trading framework for CPSs by combining blockchain, edge computing, and contract theory. Specifically, a contractbased incentive mechanism was developed to motivate EVs to participate in energy trading, and the energy trading between EVs and LEAGs is secured by exploiting consortium blockchain. Furthermore, an edge computing-based task offloading mechanism was proposed to relieve the computation burden of LEAGs and increase the successful probability of block creation. Numerical results and theoretical analysis show that the proposed framework achieves good performance in 


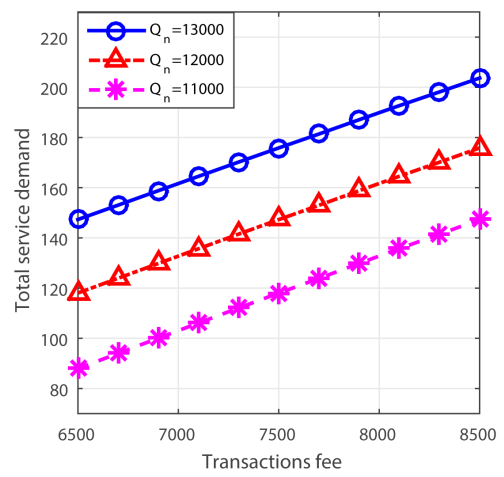

(a)

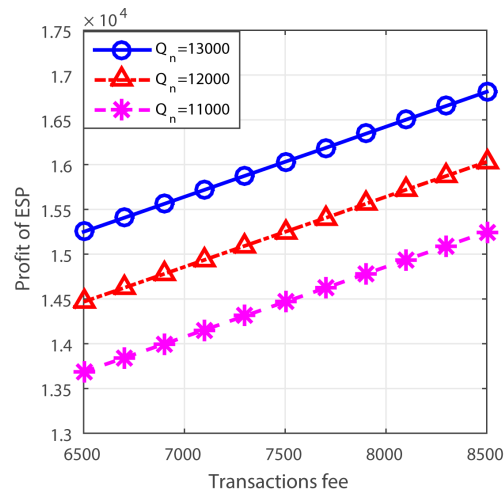

(b)

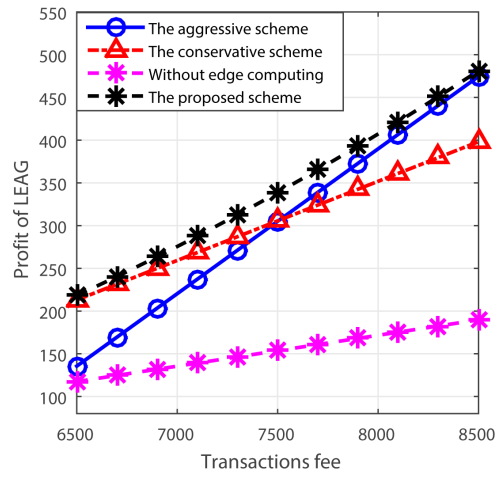

(c)

Fig. 6. Efficiency of task offloading: (a) total service demand; (b) profit of ESP; (c) profit of LEAG.

terms of contract feasibility, task offloading, and security. Some important conclusions are summarized as follows.

First, the proposed framework enables efficient energy trading under information asymmetry. The asymmetric information of EV type can be effectively elicited by the incentivecompatible contract, i.e., an EV's type is automatically revealed by observing its selection of contract items. Second, the CCP-based contract optimization algorithm can effectively maximize the expected utility of the LEAG. However, the determination of initial point has a significant impact on the convergence performance of CCP. Thus, how to select initial point to increase the convergence speed requires further investigation. Third, the successful probability of block creation can be effectively improved by utilizing edge computing. Simulation results demonstrate that the successful probability can be increased by $124.6 \%$ with the presence of eight LEAGs.

In future works, we will investigate the more complicated scenario where even the statistical knowledge of the EV type is unknown, and study how to leverage machine learning-based approaches to infer the corresponding knowledge.

\section{REFERENCES}

[1] S. A. Seshia, S. Hu, W. Li, and Q. Zhu, "Design automation of cyberphysical systems: Challenges, advances, and opportunities," IEEE Trans. Comuput. Aided Des. Integr. Circuits Syst., vol. 36, no. 9, pp. 1421-1434, Sept. 2017.

[2] J. Wu, M. Dong, K. Ota, J. Li, and Z. Guan, "FCSS: Fog computing based content-aware filtering for security services in information centric social networks," IEEE Trans. Emerg. Topics Comput., vol. PP, no. 99, pp. 1-1, Sep. 2017.

[3] C. Tham and T. Luo, "Sensing-driven energy purchasing in smart grid cyber-physical system," IEEE Trans. Syst. Man, Cybern. Syst., vol. 43, no. 4, pp. 773-784, July 2013.

[4] M. Ban, M. Shahidehpour, J. Yu, and Z. Li, "A cyber-physical energy management system and optimal sizing of networked nanogrids with battery swapping stations," IEEE Trans. Sustainable Energy, vol. PP, no. 99 , pp. $1-1$, Dec. 2017

[5] R. Deng, P. Zhuang, and H. Liang, "CCPA: Coordinated cyber-physical attacks and countermeasures in smart grid," IEEE Trans. Smart Grid, vol. 8, no. 5, pp. 2420-2430, Sept. 2017.

[6] Z. Zhou, J. Gong, Y. He, and Y. Zhang, "Software defined machine-tomachine communication for smart energy management," IEEE Commun. Mag., vol. 55, no. 10, pp. 52-60, Oct. 2017.

[7] E. Zio and G. Sansavini, "Vulnerability of smart grids with variable generation and consumption: A system of systems perspective," IEEE Trans. Syst. Man, Cybern. Syst., vol. 43, no. 3, pp. 477-487, May 2013.
[8] A. Jindal, J. S. Aujla, N. Kumar, and S. Misra, "Sustainable smart energy cyber-physical system: Can electric vehicles suffice its needs?" in Proc. IEEE International Conference Communications (ICC), Kansas City, USA, May 2018, pp. 1-6.

[9] Z. Zhou, H. Yu, C. Xu, Y. Zhang, S. Mumtaz, and J. Rodriguez, "Dependable content distribution in D2D-based cooperative vehicular networks: A big data-integrated coalition game approach," IEEE Trans. Intell. Transp. Syst., vol. 19, no. 3, pp. 953-964, Mar. 2018.

[10] A. H. Mohsenian-Rad, V. W. S. Wong, J. Jatskevich, R. Schober, and A. Leon-Garcia, "Autonomous demand-side management based on game-theoretic energy consumption scheduling for the future smart grid," IEEE Trans. Smart Grid, vol. 1, no. 3, pp. 320-331, Dec. 2010.

[11] Z. Zhou, C. Sun, R. Shi, Z. Chang, S. Zhou, and Y. Li, "Robust energy scheduling in vehicle-to-grid networks," IEEE Netw., vol. 31, no. 2, pp. 30-37, Mar. 2017.

[12] H. Li, K. Ota, and M. Dong, "Energy cooperation in battery-free wireless communications with radio frequency energy harvesting," ACM Trans. Embed. Comput. Syst., vol. 17, no. 2, pp. 44:1-44:17, Feb. 2018.

[13] E. L. Karfopoulos, K. A. Panourgias, and N. D. Hatziargyriou, "Distributed coordination of electric vehicles providing V2G regulation services," IEEE Trans. Power Syst., vol. 31, no. 4, pp. 2834-2846, July 2016.

[14] S. Pal and R. Kumar, "Electric vehicle scheduling strategy in residential demand response programs with neighbor connection," IEEE Trans. Ind. Informat., vol. 14, no. 3, pp. 980-988, Mar. 2018.

[15] A. Dorri, M. Steger, S. S. Kanhere, and R. Jurdak, "Blockchain: A distributed solution to automotive security and privacy," IEEE Commun. Mag., vol. 55, no. 12, pp. 119-125, Dec. 2017.

[16] W. L. Chin, W. Li, and H. H. Chen, "Energy big data security threats in IoT-based smart grid communications," IEEE Commun. Mag., vol. 55, no. 10, pp. 70-75, Oct. 2017.

[17] Y. Cao, T. Wang, O. Kaiwartya, G. Min, N. Ahmad, and A. Abdullah, "An EV charging management system concerning drivers' trip duration and mobility uncertainty," IEEE Trans. Syst. Man, Cybern. Syst., vol. 48, no. 4, pp. 596-607, Apr. 2018.

[18] Z. Zhou, C. Gao, Y. Zhang, S. Mumtaz, and J. Rodriguez, "Social bigdata-based content dissemination in internet of vehicles," IEEE Trans. Ind. Informat., vol. 14, no. 2, pp. 768-777, Feb. 2018.

[19] V. R. Tannahill, D. Sutanto, K. M. Muttaqi, and M. A. Masrur, "Future vision for reduction of range anxiety by using an improved state of charge estimation algorithm for electric vehicle batteries implemented with low-cost microcontrollers," IET Electrical Systems in Transportation, vol. 5, no. 1, pp. 24-32, Feb. 2015.

[20] Y. Wu, X. Tan, L. Qian, D. H. K. Tsang, W. Z. Song, and L. Yu, "Optimal pricing and energy scheduling for hybrid energy trading market in future smart grid," IEEE Trans. Ind. Informat., vol. 11, no. 6, pp. 1585-1596, Dec. 2015.

[21] H. Li, K. Ota, and M. Dong, "Learning IoT in edge: Deep learning for the internet of things with edge computing," IEEE Netw., vol. 32, no. 1, pp. 96-101, Jan.-Feb. 2018.

[22] J. Xu, K. Ota, and M. Dong, "Saving energy on the edge: In-memory caching for multi-tier heterogeneous networks," IEEE Commun. Mag., vol. 56, no. 5, pp. 102-107, May 2018. 
[23] L. Li, K. Ota, and M. Dong, "DeepNFV: A light-weight framework for intelligent edge network functions virtualization," IEEE Netw., vol. PP, no. 99, pp. 1-6, Aug. 2018.

[24] Z. Zhou, X. Fei, B. Huang, C. Xu, R. Jiao, and B. L. et al., "Gametheoretical energy management for energy Internet with big data-based renewable power forecasting," IEEE Access, vol. 5, no. 99, pp. 57315746, Feb. 2017.

[25] Z. Xiong, S. Feng, D. Niyato, P. Wang, and Z. Han, "Edge computing resource management and pricing for mobile blockchain," Comput. Research Repository, 2017. [Online]. Available: https://arxiv.org/abs/1711.01049

[26] V. Gatteschi, F. Lamberti, C. D. C. Pranteda, and V. Santamaría, "To blockchain or not to blockchain: That is the question," IT Prof., vol. 20, no. 2, pp. 62-74, Apr. 2018.

[27] C. Liu, K. K. Chai, X. Zhang, E. T. Lau, and Y. Chen, "Adaptive blockchain-based electric vehicle participation scheme in smart grid platform," IEEE Access, vol. 6, no. 99, pp. 25657-25665, May 2018.

[28] Z. Li, J. Kang, R. Yu, D. Ye, Q. Deng, and Y. Zhang, "Consortium blockchain for secure energy trading in industrial Internet of things," IEEE Trans. Ind. Informat., vol. 14, no. 8, pp. 3690-3700, Aug. 2018.

[29] S. Aggarwal, R. Chaudhary, J. S. Aujla, A. Jindal, A. Dua, and N. Kumar, "EnergyChain: Enabling energy trading for smart homes using blockchains in smart grid ecosystem," in Proc. ACM MobiHoc Workshop Networking Cybersecurity Smart City, Los Angeles, USA, Jun. 2018, pp. 1:1-1:6.

[30] J. Kang, R. Yu, X. Huang, S. Maharjan, Y. Zhang, and E. Hossain, "Enabling localized peer-to-peer electricity trading among plug-in hybrid electric vehicles using consortium blockchains," IEEE Trans. Ind. Informat., vol. 13, no. 6, pp. 3154-3164, Dec. 2017

[31] D. Sahinel, C. Akpolat, M. A. Khan, F. Sivrikaya, and S. Albayrak, "Beyond 5G vision for iolite community," IEEE Commun. Mag., vol. 55, no. 1, pp. 41-47, Jan. 2017.

[32] S. Garg, A. Singh, S. Batra, N. Kumar, and L. Yang, "UAV-empowered edge computing environment for cyber-threat detection in smart vehicles," IEEE Netw., vol. 32, no. 3, pp. 42-51, Jun. 2018.

[33] Z. Zhou, J. Feng, L. Tan, Y. He, and J. Gong, "An air-ground integration approach for mobile edge computing in IoT," IEEE Commun. Mag., vol. 56, no. 8, pp. 40-47, Aug. 2018.

[34] Z. Zhou, H. Liao, B. Gu, K. M. S. Huq, S. Mumtaz, and J. Rodriguez, "Robust mobile crowd sensing: When deep learning meets edge computing," IEEE Netw., vol. 32, no. 4, pp. 54-60, Aug. 2018.

[35] Y. Li, T. Zhao, C. H. Liu, Y. Zhao, P. Wang, H. B. Gooi, K. Li, and $Z$. Ding, "An interactive decision making model based on energy and reserve for electric vehicles and power grid using generalized Stackelberg game," IEEE Trans. Ind. Appl., vol. PP, no. 99, pp. 1-1, Sep. 2018.

[36] M. Ghofrani, A. Arabali, M. Etezadi-Amoli, and M. S. Fadali, "Smart scheduling and cost-benefit analysis of grid-enabled electric vehicles for wind power integration," IEEE Trans. Smart Grid, vol. 5, no. 5, pp. 2306-2311, Sept. 2014

[37] L. Duan, L. Gao, and J. Huang, "Cooperative spectrum sharing: A contract-based approach," IEEE Trans. Mobile Comput., vol. 13, no. 1, pp. 174-187, Jan. 2014.

[38] Y. Zhang, L. Song, W. Saad, Z. Dawy, and Z. Han, "Contract-based incentive mechanisms for device-to-device communications in cellular networks," IEEE J. Sel. Areas Commun., vol. 33, no. 10, pp. 2144-2155, Oct. 2015

[39] T. Liu, J. Li, F. Shu, M. Tao, W. Chen, and Z. Han, "Design of contractbased trading mechanism for a small-cell caching system," IEEE Trans. Wireless Commun., vol. 16, no. 10, pp. 6602-6617, Oct. 2017.

[40] Q. Wang, X. Li, and Y. Yu, "Anonymity for bitcoin from secure escrow address," IEEE Access, vol. 6, no. 99, pp. 12 336-12 341, Dec. 2017.

[41] D. Johnson, A. Menezes, and S. Vanstone, "The elliptic curve digital signature algorithm (ECDSA)," Int. J. Inf. Secur., vol. 1, no. 1, pp. 36 63, Aug. 2001.

[42] Y. Gao, X. Chen, Y. Chen, Y. Sun, X. Niu, and Y. Yang, "A secure cryptocurrency scheme based on post-quantum blockchain," IEEE Access, vol. 6, no. 99, pp. 27 205-27213, Apr. 2018

[43] W. Yin, Q. Wen, W. Li, H. Zhang, and Z. Jin, "An anti-quantum transaction authentication approach in blockchain," IEEE Access, vol. 6, no. 99, pp. 5394-5401, Jan. 2018.

[44] P. R. Rizun, "A transaction fee market exists without a block size limit," Block Size Limit Debate Working Paper, Aug. 2015. [Online]. Available: https://www.bitcoinunlimited.info/resources/feemarket.pdf

[45] D. He, N. Kumar, S. Zeadally, and H. Wang, "Certificateless provable data possession scheme for cloud-based smart grid data management systems," IEEE Trans. Ind. Informat., vol. 14, no. 3, pp. 1232-1241, Mar. 2018.

[46] D. Khovratovich, C. Rechberger, and A. Savelieva, "Bicliques for preimages: Attacks on skein-512 and the SHA-2 family," in Proc. International Conference FAST Software Encryption, Washington, DC USA, Mar. 2012, pp. 244-263.

[47] R. Anderson, Security Engineering: A Guide to Building Dependable Distributed Systems. Hoboken, New Jersey, USA: Wiley, 2001.

[48] Y. Wang and J. Gao, "A regulation scheme based on the ciphertextpolicy hierarchical attribute-based encryption in bitcoin system," IEEE Access, vol. 6, no. 99, pp. 16267-16278, Mar. 2018.

[49] Y. Zheng and J. Seberry, "Immunizing public key cryptosystems against chosen ciphertext attacks," IEEE J. Sel. Areas Commun., vol. 11, no. 5, pp. 715-724, Jun. 1993.

[50] M. Abdalla, F. Benhamouda, and D. Pointcheval, "Public-key encryption indistinguishable under plaintext-checkable attacks," IET Inf. Security, vol. 10, no. 6, pp. 288-303, Nov. 2016.

[51] S. Nakamoto, "Bitcoin: A peer-to-peer electronic cash system," 2008 [Online]. Available: https://bitcoin.org/en/

[52] G. C. Hsieh, L. R. Chen, and K. S. Huang, "Fuzzy-controlled liion battery charge system with active state-of-charge controller," IEEE Trans. Ind. Electron., vol. 48, no. 3, pp. 585-593, June 2001.

[53] T. Lipp and S. Boyd, "Variations and extension of the convex-concave procedure," Optim. Eng., vol. 17, no. 2, pp. 263-287, June 2016.

[54] N. Houy, "The bitcoin mining game," Working Paper, Mar. 2014. [Online]. Available: https://ssrn.com/abstract $=2407834$

[55] M. J. Osborne and A. Rubinstein, A Course in Game Theory. Cambridge, MA, USA: MIT Press, 1994.

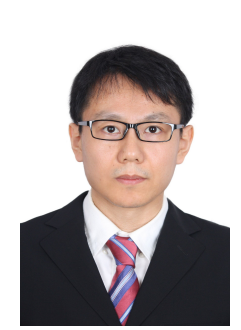

Zhenyu Zhou (M'11-SM'17) received his M.E. and $\mathrm{Ph} . \mathrm{D}$ degree from Waseda University, Tokyo, Japan in 2008 and 2011 respectively. From April 2012 to March 2013, he was the chief researcher at Department of Technology, KDDI, Tokyo, Japan. From March 2013 to now, he is an Associate Professor at School of Electrical and Electronic Engineering, North China Electric Power University, China. He served as an Associate Editor for IEEE Access, EURASIP Journal on Wireless Communications and Networking and a Guest Editor for IEEE Communications Magazine and Transactions on Emerging Telecommunications Technologies. He also served as workshop co-chair for IEEE Globecom 2018, IEEE ISADS 2015, and TPC member for IEEE Globecom, IEEE CCNC, IEEE ICC, IEEE APCC, IEEE VTC, IEEE Africon, etc. He is a voting member of IEEE Standard Association P1932.1 Working Group. He was the recipient of the IEEE Vehicular Technology Society "Young Researcher Encouragement Award" in 2009, the "Beijing Outstanding Young Talent Award in 2016, the IET Premium Award in 2017, and the IEEE ComSoc Green Communications and Computing Technical Committee 2017 Best Paper Award. His research interests include green communications, vehicular communications, and smart grid communications. He is a senior member of IEEE.

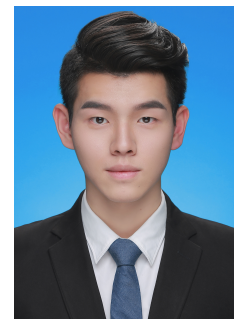

Bingchen Wang is currently working towards the B.S. degree at North China Electric Power University, China. His research interests include green communications and smart grid. 


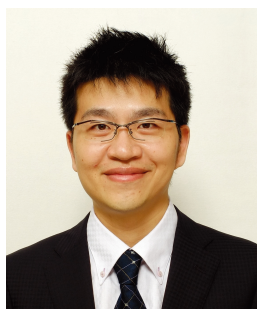

Mianxiong Dong received B.S., M.S. and Ph.D. in Computer Science and Engineering from The University of Aizu, Japan. He is currently an Associate Professor in the Department of Information and Electronic Engineering at the Muroran Institute of Technology, Japan. He was a JSPS Research Fellow with School of Computer Science and Engineering, The University of Aizu, Japan and was a visiting scholar with BBCR group at University of Waterloo, Canada supported by JSPS Excellent Young Researcher Overseas Visit Program from April 2010 to August 2011. Dr. Dong was selected as a Foreigner Research Fellow (a total of 3 recipients all over Japan) by NEC C\&C Foundation in 2011. His research interests include Wireless Networks, Cloud Computing, and Cyberphysical Systems. He has received best paper awards from IEEE HPCC 2008, IEEE ICESS 2008, ICA3PP 2014, GPC 2015, IEEE DASC 2015, IEEE VTC 2016-Fall, FCST 2017, 2017 IET Communications Premium Award and IEEE ComSoc CSIM Best Conference Paper Award 2018. Dr. Dong serves as an Editor for IEEE Transactions on Green Communications and Networking (TGCN), IEEE Communications Surveys and Tutorials, IEEE Network, IEEE Wireless Communications Letters, IEEE Cloud Computing, IEEE Access, as well as a leading guest editor for ACM Transactions on Multimedia Computing, Communications and Applications (TOMM), IEEE Transactions on Emerging Topics in Computing (TETC), IEEE Transactions on Computational Social Systems (TCSS). He has been serving as the Vice Chair of IEEE Communications Society Asia/Pacific Region Information Services Committee and Meetings and Conference Committee, Leading Symposium Chair of IEEE ICC 2019, Student Travel Grants Chair of IEEE GLOBECOM 2019, and Symposium Chair of IEEE GLOBECOM 2016 , 2017. He is the recipient of IEEE TCSC Early Career Award 2016, IEEE SCSTC Outstanding Young Researcher Award 2017, The 12th IEEE ComSoc Asia-Pacific Young Researcher Award 2017, Funai Research Award 2018 and NISTEP Researcher 2018 (one of only 11 people in Japan) in recognition of significant contributions in science and technology. He is currently the Member of Board of Governors and Chair of Student Fellowship Committee of IEEE Vehicular Technology Society, and Treasurer of IEEE ComSoc Japan Joint Sections Chapter.

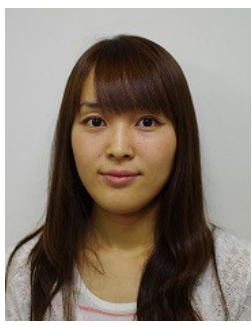

Kaoru Ota was born in Aizu-Wakamatsu, Japan. She received M.S. degree in Computer Science from Oklahoma State University, USA in 2008, B.S. and $\mathrm{Ph} . \mathrm{D}$. degrees in Computer Science and Engineering from The University of Aizu, Japan in 2006, 2012. respectively. She is currently an Assistant Professor with Department of Information and Electronic Engineering, Muroran Institute of Technology, Japan. From March 2010 to March 2011, she was a visiting scholar at University of Waterloo, Canada. Also she was a Japan Society of the Promotion of Science (JSPS) research fellow with Kato-Nishiyama Lab at Graduate School of Information Sciences at Tohoku University, Japan from April 2012 to April 2013. Her research interests include Wireless Networks, Cloud Computing, and Cyber-physical Systems. Dr. Ota has received best paper awards from ICA3PP 2014, GPC 2015, IEEE DASC 2015, IEEE VTC 2016-Fall, FCST 2017, 2017 IET Communications Premium Award and IEEE ComSoc CSIM Best Conference Paper Award 2018. She is an editor of IEEE Transactions on Vehicular Technology (TVT), IEEE Communications Letters, Peer-toPeer Networking and Applications (Springer), Ad Hoc \& Sensor Wireless Networks, International Journal of Embedded Systems (Inderscience) and Smart Technologies for Emergency Response \& Disaster Management (IGI Global), as well as a guest editor of ACM Transactions on Multimedia Computing, Communications and Applications (leading), IEEE Internet of Things Journal, IEEE Communications Magazine, IEEE Network, IEEE Wireless Communications, IEEE Access, IEICE Transactions on Information and Systems, and Ad Hoc \& Sensor Wireless Networks (Old City Publishing). She is the recipient of IEEE TCSC Early Career Award 2017, and The 13th IEEE ComSoc Asia-Pacific Young Researcher Award 2018. 